\title{
Shuttle-era experiments in the area of plasma flow interactions with bodies in space
}

\author{
URI SAMIR \\ Space Physics Research Laboratory, University of Michigan, Ann Arbor, MI 48109, U.S.A. \\ and \\ Department of Geophysics and Planetary Sciences, Tel Aviv University, Ramat Aviv, Israel
}

AND

NOBIE H. STONE

Space Science Laboratory, Marshall Space Flight Center, Huntsville, AL 35812, U.S.A.

(Received 15 July 1979)

\begin{abstract}
A new experimental approach is discussed in general terms, that can be adopted in the Shuttle/Spacelab era starting in the 1980 s for studies in the area of plasma flow interactions with bodies in space. The potential use of the Space Shuttle/Orbiter as a near Earth plasma laboratory for studies in the area of Space Plasma Physics and particularly in the area of Solar-System Plasmas is discussed. This new experimental approach holds great promise for studies in the Supersonic and sub-Alfvenic flow regime which has applications to the motion of natural satellites around their mother planets in the Solar-system (e.g. the satellite Io around the planet Jupiter). A well conceived experimental and theoretical program, can lead to a better physical understanding regarding the validity and range of applicability of using gas-dynamic, kinetic and fluid approaches in describing collisionless plasma flow interactions with bodies in a variety of flow regimes.

In addition to the above scientific aspects of the program, significant technological advances can be achieved regarding the interaction of space probes in planetary atmospheres/ionospheres and the reliability of using various plasma diagnostic devices on board spacecraft and large space platforms.
\end{abstract}

\section{Introduction-General background}

ThE ADVENT of the Shuttle/Spacelab provides a long awaited opportunity to perform in situ studies of the interactions which occur between highly rarefied plasmas and objects in space. It is therefore desirable to develop an evolutionary experimental and theoretical program of plasma flow interaction studies that will utilize the wide range of capabilities that the Shuttle/Spacelab platform offers.

The general area of "rarefied plasma flow interactions with bodies in space" deals with the complex of phenomena and cause and effect relationships involved in the creation of shocks, sharp gradients in charged particle densities, potential wells, plasma oscillations, wave-particle interactions, instabilities and plasma turbulence which may occur in regions of disturbed plasma flow surrounding a rapidly moving body in space.

Through the past decade various phenomena connected with the interaction of an object moving in the Earth's ionosphere have been investigated theoretic- 
ally and to a much lesser extent experimentally. $\dagger$ However, the information available at present, both theoretical and from in situ measurements, is insufficient to provide for an adequate examination of the physical processes involved in such interactions. It is with the advent of the Shuttle/Spacelab era that it becomes possible to use the terrestrial ionosphere-magnetosphere as a working rarefied plasma and the Shuttle/Spacelab as a laboratory suitable for performing controlled experiments covering a wide scope of scientific objectives.

During the last decade, several in situ investigations whose major scientific objective was to study the angular distribution of charged particles in the near vicinity of ionospheric satellites were undertaken. $\ddagger$ Unfortunately, these studies were limited in scope and fragmentary in nature and should therefore be seen as exploratory. Only small samples of in situ measurements lent themselves to partial theory-experiment comparisons. $\$$

In addition to the interest in this area of investigation from the pure science point of view, in particular for the supersonic and sub-Alfvenic plasma flow regime, it should be obvious that the understanding of phenomena involved in the interaction between a satellite and the terrestrial ionosphere (or: "wakes and sheaths around satellites" as this area of research is often called in its narrower sense) is needed for the better interpretation of in situ measurements of thermal ions and electrons and local field potentials. In fact, there can be little doubt that the disturbances created by the motion of the satellite have profound relevance to the reliability and quality of the measurements made by instruments on board, Spacelab and other large space platforms.

The theoretical study of the above problem requires the self-consistent solution of the Vlasov-Poisson equations (for ions and electrons) under realistic boundary conditions. This is a difficult mathematical task which therefore calls upon simplifying assumptions to be adopted. Unfortunately, such assumptions are not always in accord with the physical situations which appear in nature. The interaction between a body (in space) and a plasma is mutual and the phenomena involved are coupled by effects on both the satellite and the plasma. It is therefore reasonable to assume that theory-experiment comparisons could aid in assessing the value of various simplifying assumptions used in the theoretical studies for specific plasma flow regimes. However, the incomplete nature of the available in situ observations (Samir, 1973a; Al'pert, 1976) do not allow for such comparisons to be made in a comprehensive and meaningful manner. Also, it should be further realized that, since most available direct measurement results come from probes that are flush mounted on spacecraft surfaces, no direct information can be obtained for regions which are not very close to the surface. Hence, specific structural characteristics as shocks, density and temperature

†See Gurevich et al., 1970; Al'pert, 1976; Samir, 1973; and Liu, 1969, 1975.

¥See Samir, 1973; Troy et al., 1975; Samir et al., 1975; Vernet-Mayer, 1976; and Samir et al., $1979 \mathrm{a}, \mathrm{b}$.

\$Gurevich et al., 1970; Samir and Jew, 1972; Weil et al., 1973; Dimant and Gurevich, 1975; and Samir et al., 1979a, b. 
enhancements and depletions, plasma oscillations, etc. do not (a priori) lend themselves to direct measurements made by such probes.

In its most general context, the area of plasma flow interactions also encompasses many natural phenomena, for example, the interaction of the solar wind with the planets.

No serious attempt has ever been made to use artificial satellites moving in the ionosphere as "a model" for understanding physical processes involved in rafefied plasma flow interactions with objects in space (e.g. the Moon, planets with no intrinsic magnetic field, etc.) in this more general context. Recently, attempts were made to assess the possibility of using the principle of "qualitative scaling" (Falthammar, 1974) for "model" experiments to be performed on board Spacelab (Samir, 1976; Stone and Samir, 1980). The study of phenomena and physical processes involved in body-plasma interactions should yield an improved physical insight into the degree of applicability of gas-dynamic, plasma kinetic and fluid approaches in describing the interactions of collisionless plasma flows with a variety of bodies in space. Such approaches are often used to describe the interactions of the solar wind with planets and their satellites as well as with comets and artificial satellites moving in planetary environments (Sprieter, 1976; Perez-deTejana et al., 1977; Block, 1976). The question of when and why is there a dualism in the description of the above interactions using microscopic and/or macroscopic approaches is not always physically clear. The latter point was discussed in a variety of contexts. $\dagger$

In addition to the purely theoretical work in this area and the in situ experimental effort mentioned above quite a significant amount of experimental work was done in the laboratory. The laboratory work focused on attempts to simulate some aspect of the interaction between a satellite and the terrestrial ionosphere (Fournier and Pigache, 1975, 1977; Samir et al., 1974a and b; Stone et al., 1978) and flow around test bodies with potential applications to the flow of the solar wind around planetary objects (Podgorny, 1976; Falthammar, 1974; Block, 1976).

However, the use of such laboratory work is often subject to controversy due to the technological difficulties involved in achieving exact scaling relationships of relevance to space applications. On the other hand the obvious advantage that the laboratory simulation experiments have over the past decade of in situ experiments lies in the element of CONTROL over the entire experiment and the possibility to map wide regions of the flow field around adequately selected "tests bodies" which interact with a synthetic streaming laboratory plasma. In order to achieve such a comprehensive mapping in space one has to utilize a multi-satellite system. Results from such an investigation have not been reported in open literature in the last decade. An alternative to such an experimental approach is to use the extensive range of capabilities offered by the Shuttle/Spacelab system. In particular the capability of ejecting subsatellites and the use of maneuverable booms mounted on board the Spacelab platform.

†See Sprieter and Rizzi, 1972; Wallis, 1973; Cloutier, 1976; Lipatov, 1976; and Belotserkovskii and Mitnitskii, 1976. 
It becomes clear that the advent of the Space Shuttle/Spacelab allows workers in the field of "Rarefied Plasma Flow Interactions with Objects in Space" to adopt an experimental approach which combines the experience and methods employed in space with the practices of controlled experimentation used in laboratory plasma chanbers (or plasma wind tunnels). The Spacelab can therefore be seen as a rarefied plasma laboratory which permits the plasma physicist, rarefied gas dynamicist and the experimental astrophysicist access to plasma regions whose properties approach the assumptions often made in plasma theory (e.g. of an infinite, homogenous fully ionized collisionless magnetoplasma).

\section{An experimental philosophy for the Shuttle/Spacelab era-general}

The experimental philosophy of the Shuttle era is based upon using the Spacelab as a near Earth plasma laboratory where controlled experiments can be performed using the ionosphere-magnetosphere as the "working" rarefied plasma. It is known that the flow field characteristics around bodies in space depend on parameters such as:

$$
R_{D}=R_{0} / \lambda_{D}, \quad S=\frac{V_{s}}{\sqrt{\frac{2 k T_{e}}{M+}}} ; \quad T=\frac{T_{e}}{T_{i}} ; \quad \phi_{N}=\frac{l \phi}{k T_{e}} ; \quad R_{L}(e)=\frac{R_{0}}{\delta e} ; \quad R_{L}(+)=\frac{R_{0}}{\delta+}
$$

where $R_{D}=$ normalized linear dimension, $s=$ ionic Mach number, $T=$ ratio of electron to ion temperature, $\phi_{N}=$ normalized potential, $R_{L}(e)$ and $R_{L}(+)$ ratios of effective radius to electron and ion Lermor radii $\delta_{e}$ and $\delta_{+}$respectively. And, $R_{0}=$ effective radius of the body, $\lambda_{D}=$ Debye length, $\phi=$ body and/or local field potential. It will therefore be very useful to measure charged particle properties throughout an extensive spatial region around the body over a large range of the above parameters. Tables 1-3 provide some nominal values of relevant ambient plasma properties and parameters in the altitude range $200-3000 \mathrm{~km}$ (after: Boyd, 1968).

In order to investigate the influence of the parameter $R_{D}$ on some prominent structural characteristics (e.g. trailing shocks, density and temperature

Table 1. Some nominal values of plasm--properties in the near-Earth environment

\begin{tabular}{lccccc}
\hline$H(\mathrm{~km})$ & 200 & 400 & 800 & 1200 & 3000 \\
\hline$B($ gauss $)$ & 0.5 & 0.5 & 0.4 & 0.3 & 0.2 \\
$N_{0}\left(\mathrm{~cm}^{-3}\right)$ & $8 \times 10^{9}$ & $2 \times 10^{8}$ & $2 \times 10^{6}$ & $10^{5}$ & $10^{4}$ \\
$N e,+\left(\mathrm{cm}^{-3}\right)$ & $4 \times 10^{5}$ & $10^{6}$ & $10^{5}$ & $10^{4}$ & $5 \times 10^{3}$ \\
$T_{N}(\mathrm{~K})$ & $1.1 \times 10^{3}$ & $1.4 \times 10^{3}$ & $1.4 \times 10^{3}$ & $1.4 \times 10^{3}$ & \\
$T_{e}(\mathrm{~K})$ & $2 \times 10^{3}$ & $2.8 \times 10^{3}$ & $3 \times 10^{3}$ & $(3-4) \times 10^{3}$ & $5 \times 10^{3}$ \\
$T_{+}(\mathrm{K})$ & $1.1 \times 10^{3}$ & $1.6 \times 10^{3}$ & $2.2 \times 10^{3}$ & $2.6 \times 10^{3}$ & \\
$M_{+}($a.m.u. $)$ & 24 & 20 & 14 & 10 & 2 \\
Major ions & $\mathrm{NO}^{+}, \mathrm{O}^{+}$ & $\mathrm{NO}^{+}, \mathrm{O}^{+}$ & $\mathrm{O}^{+}, \mathrm{H}_{e}^{+}, \mathrm{H}^{+}$ & $\mathrm{O}^{+}, \mathrm{H}_{\mathrm{e}}^{+}, \mathrm{H}^{+}$ & $\mathrm{H}_{\mathrm{e}}^{+}, \mathrm{H}^{+}$ \\
\hline
\end{tabular}

After Boyd, 1968. 
Table 2. Some nominal values of plasma-parameters in the near-Earth environment

\begin{tabular}{lccccc}
\hline \multicolumn{1}{c}{$H(\mathrm{~km})$} & 200 & 400 & 800 & 1200 & 3000 \\
\hline$L e,+(\mathrm{cm})$ & $1 \times 10^{5}$ & $1 \times 10^{5}$ & $1 \times 10^{6}$ & $1 \times 10^{7}$ & $3 \times 10^{7}$ \\
$V_{T}(+)(\mathrm{cm} / \mathrm{sec})$ & $1 \times 10^{5}$ & $1.4 \times 10^{5}$ & $1.8 \times 10^{5}$ & $2.4 \times 10^{5}$ & $7 \times 10^{5}$ \\
$V_{T}(e)(\mathrm{cm} / \mathrm{sec})$ & $3 \times 10^{7}$ & $3 \times 10^{7}$ & $3 \times 10^{7}$ & $3 \times 10^{7}$ & $4 \times 10^{7}$ \\
$\delta+(\mathrm{cm})$ & $5 \times 10^{2}$ & $5 \times 10^{2}$ & $7 \times 10^{2}$ & $8 \times 10^{2}$ & $8 \times 10^{2}$ \\
$\delta e(\mathrm{~cm})$ & 3 & 3 & 4 & 6 & 11 \\
$\partial e,+\left(\mathrm{sec}^{-1}\right)$ & $2 \times 10^{2}$ & $3 \times 10^{2}$ & $3 \times 10^{1}$ & 4 & 0.2 \\
$\omega+\left(\mathrm{sec}^{-1}\right)$ & $2 \times 10^{2}$ & $2 \times 10^{2}$ & $3 \times 10^{2}$ & $3 \times 10^{2}$ & $10^{3}$ \\
$\omega e\left(\mathrm{sec}^{-1}\right)$ & $9 \times 10^{6}$ & $9 \times 10^{6}$ & $7 \times 10^{6}$ & $5 \times 10^{6}$ & $4 \times 10^{6}$ \\
$\lambda_{D}(\mathrm{~cm})$ & 0.5 & 0.4 & 1.2 & 4.1 & 7 \\
\hline
\end{tabular}

Table 3. Nominal values for some useful parameters relevant to spacecraftionosphere interactions. (For: $R_{0}=50 \mathrm{~cm} ; R(p)=5 \mathrm{~cm} ; V_{S}=7.5 \times$ $10^{5} \mathrm{~cm} / \mathrm{sec}$ )

\begin{tabular}{lccccc}
\hline \multicolumn{1}{c}{$H(\mathrm{~km})$} & 200 & 400 & 800 & 1200 & 3000 \\
\hline$L e,+/ R_{0}$ & $2 \times 10^{3}$ & $2 \times 10^{3}$ & $2 \times 10^{4}$ & $2 \times 10^{5}$ & $6 \times 10^{5}$ \\
$R_{D}=\left(R_{0} / \lambda_{D}\right)$ & 100.0 & 125.0 & 41.7 & 12.2 & 7.1 \\
$R_{P}=R(p) / \lambda_{D}$ & 10 & 12.5 & 4.2 & 1.2 & 0.7 \\
$R_{L}(+)=R_{0} / \delta+$ & 0.1 & 0.1 & 0.07 & 0.06 & 0.06 \\
$R_{L}(e)=R_{0} / \delta e$ & 16.7 & 16.7 & 12.5 & 8.3 & 4.5 \\
$T=T d T_{+}$ & 1.8 & 1.7 & 1.4 & 1.3 & 1.0 \\
$S=\frac{V_{S}}{V_{T}(+)}$ & 7.5 & 5.4 & 4.2 & 1.7 & 1.1 \\
\hline
\end{tabular}

where:

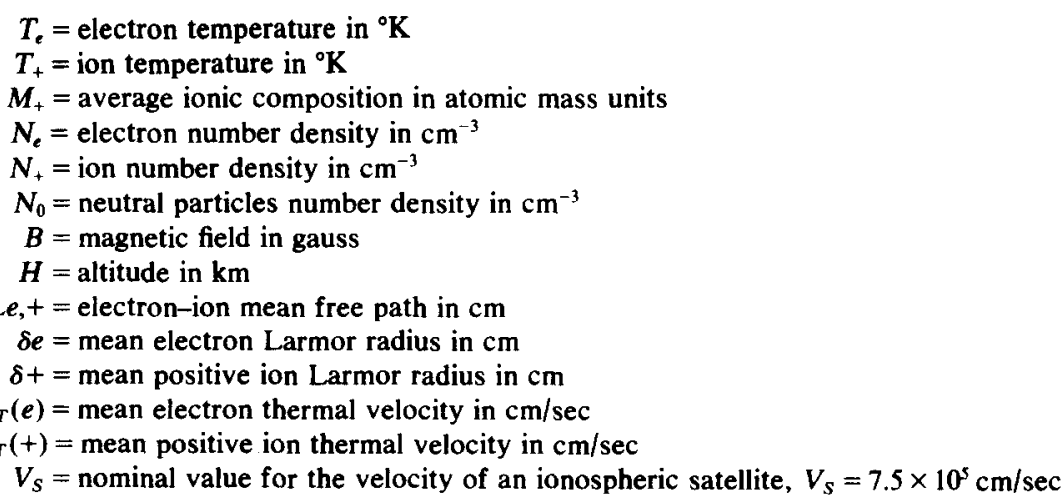




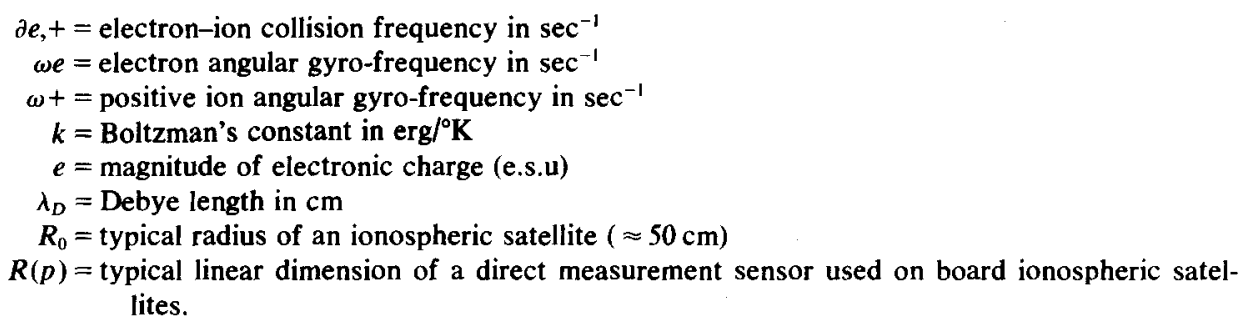

enhancements and depletions) of the flow fields around a rapidly moving body in space it will be necessary to use bodies of different sizes, moving in plasmas with different values of density $\left(N_{e}\right)$ and temperature $\left(T_{e}\right)$. Utilizing the wide range of capabilities of the Shuttle/Spacelab system, a variety of "test bodies" could be mounted on booms and exposed to the natural plasma flow. Direct measurement devices (e.g. Langmiur probes, Retarding Potential Analyzers, Faraday cups, Mass Spectrometers, electric field measuring devices such as spheres on relatively short booms, magnetometers, etc.) could then be packaged together and mounted on another articulated and maneuverable boom mounted on Spacelab. Depending on the degree of maneuverability of the booms, fairly wide spatial regions around relatively small and intermediate (say of $R_{0} \sim$ $100 \mathrm{~cm}$ ) "test bodies" can be mapped (Samir et al., 1977).

The capability of launching or ejecting small bodies from the Spacelab itself is of great value. Such ejected bodies, often referred to as: throw away detectors (TADs) and subsatellites (of various degrees of sophistication) can be made to orbit around pre-selected and appropriately designed large "test bodies" [say, of $R_{0} \sim(10-100) \mathrm{m}$ ]. The "test bodies" themselves can be both rigid bodies and/or inflatable balloons, and can be either instrumented or non-instrumented. The latter group of "test bodies" can therefore act as both "objects" whose flow fields are to be investigated and as diagnostic TADs and/or subsatellites.

Tethered bodies of different sizes and with a large range of "wire" length can also be used. Numerous combinations employing controlled "test bodies" and diagnostic ensembles exist; the preferred arrangement depending on the specific scientific and technology objectives sought for particular missions. The flexibility expected to be available in using "test bodies" allows for the use of bodies having intrinsic magnetic fields and capable of being oriented at various and pre-selected inclinations to the flow vector and to the geomagnetic field. Information from such experiments could be useful to Astrophysics and Cosmic Physics. In order to achieve the latter (a controversial subject at the present time) the concepts of "process simulation" and "qualitative scaling" will have to be used. This will be discussed in some detail later.

Another parameter of great significance in determining the prominent characteristics of the flow field structure around bodies in space is the potential on the body normalized by the energy of the electrons; i.e. $\phi_{N}=\left(e \phi_{B} / K T_{e}\right)$ where $\phi_{B}$ is the body potential obtained from $\Sigma I=0$ and where $\Sigma I$ denotes the 
total current to the body. The in situ measurements performed in the last decade regarding $\phi_{B}$ were prescribed by particle collection from the natural environment of the body. As is well known, the realistic significance of the various charging mechanisms which contribute to $\phi_{B}$ for various space plasmas is still an open subject and "spacecraft charging" is at present an active area of theoretical and experimental research, particularly with respect to its applications to ionospheric and magnetospheric satellites (Pike and Lovell, 1977; Rosen, 1976).

The vast Space Shuttle/Spacelab capabilities allow us to bias the "test bodies" in a manner suitable for parametric examination. We emphasize that such a capability was not available for in situ observations in the pre-Shuttle era. Biasing of "test bodies" in synthetic plasmas is of course possible and was done throughout the last decade in laboratory simulation studies. $\dagger$ In order to "map" to flow fields around the test bodies the diagnostic devices should be able to measure the local energy distributions of ions and electrons their densities and local electric and magnetic fields. It is not very difficult to design a program that should progress in an evolutionary manner starting with fairly simple but comprehensive field mappings around small/intermediate size bodies and progressing toward large bodies $\left(R_{0} \approx 10-100 \mathrm{~m}\right)$ which can be tethered balloons, large spheres/cylinders, etc. mounted on long booms and employing the facility for ejecting throw away detectors (TADs) and subsatellites. The general discussion given above deals with the utilization of the Shuttle/Spacelab system, including ejectable bodies and subsatellites, for in situ measurements relevant to the general area of "Rarefied Plasma Flow Interactions with Bodies in Space". The discussion that follows is restricted to (1) the performing of experiments that may have astrophysical or cosmic physical (or: space physics) applications, and (2) the performing of experiments which are directly relevant to the study of wakes and sheaths around various bodies moving rapidly in the Earth's ionospheric-magnetospheric plasma, as well as in other planetary environments.

\section{Laboratory experiments of interest to space physics}

As mentioned earlier we view the Spacelab system as a near-Earth plasma laboratory where controlled experiments can be performed in a manner similar to experiments in the laboratory (i.e. plasma chambers, plasma wind tunnels). We submit that employing the concepts of process simulation and qualitive scaling should provide for a very useful (in situ) experimental program where cause and effect relationships and relevant physical mechanisms can be revealed even though the strict scaling relationships are relaxed in accordance with the definition of the concept of qualitative-scaling discussed below. Such an approach was recently used, despite skepticism by various researchers and met an unignorable degree of success (Block, 1976; Podgorny, 1976). It will therefore be useful to briefly review some of the recent laboratory simulation work and discuss some of the achievements and limitations. 1978.

†See Hester and Sonin, 1970; Fournier and Pigache, 1975; Samir et al., 1974; and Stone et al., 
The merits and limitations of laboratory studies performed in closed systems have been discussed and point towards the fundamental differences between conducting configuration simulation experiments and process simulation experiments where the principle of qualitative scaling is employed. Among the papers dealing with this dubject, we cite; Falthammar (1974), Block (1976), Podgorny (1976) and Andirjanov and Podgorny (1976).

Falthammar (1974) discusses laboratory experiments designed to study (1) solar wind interactions with self-magnetized and non-magnetized bodies, (2) neutral lines and sheaths, (3) wave-particle interactions, (4) anomalous resistivity, (5) double layers, (6) magnetic field-aligned electric fields in strong magnetic mirrors, and (7) the critical ionization velocity of a plasma moving through a neutral gas.

Podgorny (1976) and Andrijanov and Podgorny (1976) discuss laboratory simulation experiments relevant to plasma penetration into a magnetosphere and the effect of frozen-in magnetic fields on the formation of a sharp plasma boundary, e.g. as assumed for Venus. In earlier studies, they describe the flow patterns around non-conducting bodies which are relevant to the case of the Moon's wake. The experiments also deal with bodies which have an intrinsic magnetic field, as well as with bodies which do not have a significant intrinsic magnetic field but have an "atmosphere" (analogous to the case of Venus in the solar wind).

Laboratory experiments with applications to interplanetary and terrestrial phenomena were also performed by Kawashima (1971). In particular, the experiments related to energy dissipation and particle acceleration in the context of cometary simulation (Ohyabu et al., 1973). The Russian group has also performed laboratory simulation experiments of flows around comet-type bodies (Podgorny, 1976; Andrijanov and Podgorny, 1976). At the present time most of the laboratory experiments of interest to space physics (i.e. terrestrial magnetosphere, solar wind and astrophysics) are being pursued very actively at the Plasma Physics Department of The Royal Institute of Technology in Stockholm Sweden, and at the Space Research Institute at Moscow. Both groups are utilizing the concepts of process simulation via qualitative scaling quite successfully for specific problems.

It is perhaps appropriate at thie point to briefly clarify the meaning of configuration simulation, process simulation and the principle of qualitative scaling. In "configuration" or "entire system" simulation, which was the traditional type of laboratory simulation performed typically prior to the $1970 \mathrm{~s}$, the major scientific objective is the simulate the actual configuration of a system in space, for example, the whole magnetosphere. When space physics was in the era of exploration such attempts were understandable. Now that space physics has entered an era where the better understanding of physical processes and cause and effect relationships is of primary interest, it should be appropriate to attempt a simulation which focuses on clairfications of physical processes and which may sometimes be of a more basic and conceptual nature; hence the concept of process simulation.

In process simulation it is the local behaviour of a plasma that is simulated 
rather than the entire system. In this way, selected phenomena or physical processes can be studied effectively. In order to perform laboratory experiments in the process simulation mode, the principle of qualitative scaling should be used. This principle permits some relaxation of the classical Vlassov scaling laws by requiring only that (1) the relevant dimensionless parameters which are of the order of unity in space should (as far as possible) retain this value in the laboratory, and (2) the relevant dimensionless parameters which are either much larger or much smaller than unity should retain this inequality in the laboratory; however it is not necessary to preserve the inequality by the same order of magnitude.

The utility of this relazation can be appreciated by considering some of the classical Vlassov scaling laws given in Table 4. For example, it becomes immediately evident that it is not possible to simulate the entire magnetosphere in a laboratory in the configurational sense. In addition to the length factor, the magnetic fields required are of the order of $10^{8}$ gauss. However, qualitative process simulation sllows these problems to be circumvented and the success already achieved in laboratory studies of this type by both the Swedish and Russian groups should not be overlooked. Conventional wisdom and uneasiness in applying laboratory results to space should not result in a negative bias towards the further exploitation of such a promising approach. It should suffice to mention that concepts such as Birkeland currents, electric fields along magnetic field lines and electrostatic particle acceleration (Falthammar, 1974) were conceived as a result of close contact with laboratory work and, as is well known, a great effort in magnetospheric physics is now devoted to study and utilization of these processes.

Caution should, however, be exercised in claiming direct analogies to cosmic phenomena. It should also be realized that the principle of qualitative scaling can be very useful in examining phenomena for which only qualitative theory exists.

It is our opinion that, despite obvious limitations, simulation studies which utilize the principle of qualitative scaling should be seen as important to basic rarefied-plasma physics and as being useful to studies which seek a better understanding of terrestrial interplanetary and cosmic phenomena, in particular in the supersonic but sub-Alfvenic plasma flow regime.

In Podgorny (1976) the question of plasma intrusion or plasma penetration into the magnetosphere and the question of the important role of the magnetic

Table 4. Scaling laws for some plasma parameters (after Block, 1976)

\begin{tabular}{ll}
\hline $\begin{array}{l}\text { 1. } \text { Length, time, resistivity } \\
\text { 2. Particle energy, velocity, }\end{array}$ & vary as $\gamma^{+1}$ \\
$\begin{array}{l}\text { 3. Particle density and pressure, } \\
\text { electric and magnetic field }\end{array}$ & \\
frequency & vary as $\gamma^{0}$ \\
$\begin{array}{l}\text { 4. Magnetic pressure, space charge } \\
\text { density, current density }\end{array}$ & vary as $\gamma^{-2}$ \\
\hline
\end{tabular}


field frozen in the solar wind in forming the ionosphere of non-magnetic bodies was dealt with and the usefulness of the results obtained are of great interest and significance. For the case of non-magnetic bodies without a plasma shell (e.g. without an atmosphere-ionosphere) results are obtained which show similarity to what was concluded from the Explorer 35 satellite for the behavior of the magnetic field in the cavity behind the Moon, with the exception of the behaviour of the B-field along the external Mach cone. More specifically, this laboratory experiment (Podgorny, 1976) showed the absence of a disturbance on the day-side and the formation of a conic cavity with decreased density on the night-side. In the cavity, the magnetic field was more intense than in the ambient solar wind. Such a behaviour is in general accord with some conclusions based on measurements from the Explorer 35 satellite. Hence, the laboratory experiments have reproduced a number of features predicted to exist in the wake of the Moon.

The Russian Group at the Space Research Institute have also performed experiments to partially simulate the solar wind-Venus interaction, by using a model-body which evaporates an "atmosphere" upon "plasma-wind" impact.

Table 5 (after Podgorny, 1976) gives the values in space (magnetosphere) and in the laboratory for some relevant parameters under which the experiments mentioned earlier were performed. As is seen from this table the Russian Group was able to achieve qualitative scaling for all relevant parameters with the exception of the parameter $\left(\delta_{+} \equiv \rho_{+}\right)$.

It should be realised that the flow of a synthetic rarefied plasma over a non-magnetized body may, at least partially, simulate a comet, the Moon, Venus (or any other planet with an insignificant internal magnetic field), as well as a spacecraft moving in the near-Earth and planetary environments.

Dubinin et al. (1977) studied, in the laboratory, the magnetic field disturbance created by the impingement of a supermagnetisonic collision-free plasma upon a

Table 5. Values in space (magnetosphere) and in the laboratory for some relevent parameters (after Podgorny, 1976)

\begin{tabular}{|c|c|c|}
\hline Dimensionless parameters & Space & $\begin{array}{l}\text { Laboratory } \\
\text { experiments }\end{array}$ \\
\hline Magnetic Reynolds number, $\operatorname{Re}_{m}$ & $10^{12}$ & $10^{3}$ \\
\hline Mach number, $M_{s}$ & $5-10$ & $6-8$ \\
\hline Alfven's Mach number, $M_{A}$ & $4-8$ & $6-7$ \\
\hline Ratio of plasma to magnetic pressure & 1 & 3 \\
\hline $\begin{array}{l}\text { Square of ratio of Larmor to } \\
\text { Langmuir frequencies }\end{array}$ & $10^{\circ}$ & $10^{+}$ \\
\hline $\begin{array}{l}\text { Ratio of electron Larmor radius } \\
\text { to magnetosphere dimension, } \rho_{\rho} / L\end{array}$ & $5 \times 10^{-4}$ & $5 \times 10^{2}$ \\
\hline $\begin{array}{l}\text { Ratio of ion Larmor radius to } \\
\text { magnetosphere dimension, } \rho_{+} / L\end{array}$ & $10^{-2}$ & 1 \\
\hline $\begin{array}{l}\text { Ratio of Debye radius to } \\
\text { magnetospheric dimension, } \lambda_{D} / L\end{array}$ & $5 \times 10^{-6}$ & $10^{-3}$ \\
\hline $\begin{array}{l}\text { Ratio of free path length to } \\
\text { magnetosphere dimension, } \lambda / L\end{array}$ & $10^{3}$ & 10 \\
\hline
\end{tabular}


Table 6. $\dagger$ Scaling of the solar wind interaction with the Moon (after, Dubinin et al., 1977)

\begin{tabular}{lcc}
\hline \multicolumn{1}{c}{ Dimensionless parameters } & Space & Laboratory \\
\hline Magnetic Reynolds number & $10^{12}$ & $10^{3}$ \\
Mach number & $5-10$ & 3 \\
Alfvenic Mach number & 8 & $7-9$ \\
Ratio of gas-kinetic to magnetopressure & -1 & $10-20$ \\
Ratio of electron Larmor radius to obstacle size & $10^{-3}$ & $10^{-1}$ \\
Ratio of ion Larmor radius to obstacle size & $10^{-2}$ & 2 \\
Ratio of Coulomb m.f.p to obstacle size & $10^{-5}$ & 10 \\
Square of ratio of Larmor frequency and & & $10^{-5}$ \\
$\quad$ Langmuir frequency of electrons & & $10^{-6}$ \\
\hline
\end{tabular}

†Compare with Table 5.

hollow, electrically non-conducting body ("Lunella"). This study was an attempt to simulate in the laboratory some aspects of the lunar magnetosphere. The dimensionless parameters used in this study are given in Table 6 .

As seen from Table 6 the conditions in the laboratory satisfy the principle of qualitative scaling except for the ratio of the ion gyro radius to the radius of the test-body (i.e. the Lunella). Dubinin et al. (1977) state that the results obtained in this laboratory experiment are in qualitative accord with lunar magnetosphere data and bear upon the interaction of the solar wind with small non-magnetic bodies.

The Swedish group at the Royal Institute performed laboratory simulation work relevant to the terrestrial magnetosphere and to the interaction between a laboratory-produced solar-wind type plasma with a dipole (i.e. terrella-type experiments). For more details on the above experiments including the main achievements reached we cite: Falthammar (1974), Kristoferson (1975), Block and Falthammar (1976), and Block $(1975,1976)$. The prominent thing that stands out from the above studies is the degree of success achieved in using the concepts of "process-simulation" and the principle of "qualitative scaling". Block (1976) states that the virtue of process simulation can be expressed as follows: (1) New theories should as far as possible be tested in the laboratory (2) most plasma processes observed in the laboratory are probably of importance in space, (3) proper application of a laboratory process to space conditions requires a theoretical understanding of its dependence on all plasma parameters and boundary conditions.

Among other references related to the discussion of laboratory scaling rules, qualitative scaling and achievements of relevant experimental work we cite: Podgorny and Dubinin (1974), Block (1967), Dubinin and Podgorny (1974), Danielson and Kasai (1968), Podgorny et al. (1973), Podgorny and Sagdeev (1970), Schindler (1969), Schatzman and Bierman (1974), Fahleson (1967), and Olson (1974).

We submit that using the Spacelab platform as a near-Earth (in situ) plasma laboratory holds great promise for future work in space simulation since it has 
most of the advantages of laboratory work together with a natural plasma, free of artificial boundary conditions.

In addition to the model experiments relevant to flow interactions of the solar wind with planets and their moons discussed above, a considerable effort was devoted to studying (in the laboratory) various aspects of the interaction between an ionospheric satellite and its natural environment.

Among the studies in this area we cite: Samir et al. (1974a, b); Stone et al. (1974, 1978), and Oran et al. (1974, 1975); who performed work in the NASA Marshall Space Flight Center (MSFC) in the U.S.A. and Fournier and Pigache (1975), who performed work at ONERA in France. Table 7 gives the plasma flow parameters and the bodies used in the Marshall Space Flight Center wind tunnel facility. The studies were aimed at determining the general flow patterns around "test-bodies" in a streaming rarefied plasma, focusing mainly on applications to the interaction between a satellite and the terrestrial ionosphere. Details on plasma flow parameters of other facilities in the U.S.A., U.S.S.R., France and the U.K. are given elsewhere (Samir et al., 1977).

Figure 1 shows, in a schematic manner, the experimental set-up commonly used for performing such experiments in the laboratory. Most laboratories which focused on the partial simulation of the motion of a satellite in the ionosphere have tried to look for prominent features in the flow field around the bodies under differing plasma flow and body geometries. Prior to 1974 a great deal of the work was devoted to studying the ion and potential distribution around bodies in the regime $\left|R_{0} / \lambda D\right| \leqslant 1$ and relatively little work was done for the case $\left|R_{0} / \lambda D\right| \gg 1$ which is the more relevant case for satellite-ionosphere interaction. On the other hand the experimental and theoretical work for $\left|R_{0}\right| \lambda D \mid \leqslant 1$ could be applied to the case of the perturbations created by diagnostic devices on board spacecraft (Stone et al., 1978).

Although it is not the purpose of this paper to provide a detailed account of the advantages and disadvantages of the above laboratory work we should state that all suffer from the inability to simulate the ion thermal motion (Samir et al., 1977; Fournier and Pigache, 1975) which may have critical importance in understanding the physical mechanism responsible for ion density enhancement in the wake at a distance of the order of $z=\left|V s / V_{T}(+)\right| \cdot R_{0}$ (where $z$ is the axis of the wake). This problem was discussed in detail by Fournier and Pigache

Table 7. Some plasma flow parameters and "test-body" geometry used in simulation experiments (After: Samir $e t$ al., 1974a, 1977)

\begin{tabular}{lrrrr}
\hline & \multicolumn{4}{c}{ Plasma parameters } \\
\cline { 2 - 5 } Body geometry & \multicolumn{1}{c}{$\boldsymbol{R}_{D}$} & \multicolumn{1}{c}{$S$} & $\left|\phi_{N}\right|$ & $T$ \\
\hline Sphere & $1-25$ & $4-17$ & $3.5-48$ & $\ll 1$ \\
Cylinder & $12-25$ & $7-12$ & $4-48$ & $\ll 1$ \\
Square sheet & $\sim 14$ & $\sim 7.5$ & $3.5-46$ & $\ll 1$ \\
\hline
\end{tabular}




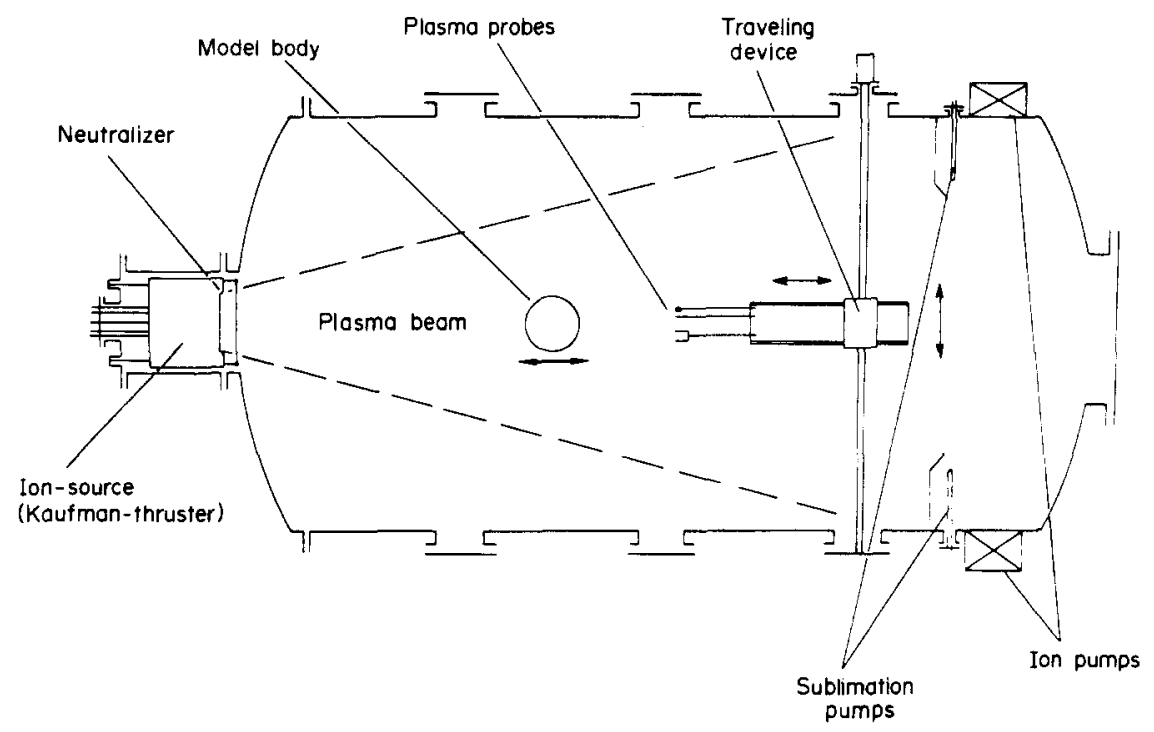

Fig. 1. Schematic drawing of the M.S.F.C. wind tunnel facility.

(1975) who focused on laboratory work and by Samir et al., (1974b) who attempted to speculate on the real importance of $V_{T}(+)$ simulation via an examination of laboratory and in situ measurements.

Among the results relevant to the knowledge of the structure of the plasma surrounding ionospheric satellites we cite: (1) ions passing through the plasma sheath are focused onto the wake axis, creating an enhancement of the ion current which peaks at a distance of about $\left|V_{S} / / V_{T}(+)\right| R_{0}$ downstream from floating spherical bodies; (2) the location of the peak enhancement exhibits a hyperbolic-type dependence on body potential; (3) the structure of the ion current enhancement seems to depend primarily on body cross section geometry rather than axial dimensions; (4) an electron temperature enhancement can sometimes occur in the near-wake zone with a magnitude inversely proportional to particle density. The physical mechanism responsible for the latter observation is still not clear. The above studies were limited to regions relatively close to the "test-bodies" for the cases where $\left(R_{0} / \lambda_{D}\right) \gg 1$. Performing experiments from the Spacelab (operating as a near-Earth plasma laboratory) will remove the above severe limitation as well as remove the limitation of the correct simulation of $V_{T}(+)$. More details on relevant laboratory work are given in: Stone et al. (1974), Samir et al. (1974a), Martin (1974), and Stone et al. (1978).

\section{About satellite-ionospheric interactions}

In this section the present state of knowledge of the phenomena involved in the interaction between a satellite and the terrestrial ionosphere is discussed. Since the Shuttle/Spacelab is to orbit in the terrestrial ionosphere it is expected that the analysis given in this section will be useful for both science and technology purposes. It should be kept in mind that the satellite-ionospheric 
interaction is a special case of the general area of "plasma flow interactions with objects in space" discussed earlier.

The discussion in this section is divided into: (1) a general theoretical discussion of assumptions (physical and mathematical) used in the various wake and sheath models which are relevant to ionospheric satellites, and (2) an examination of the most outstanding results obtained in the last decade from in situ measurements.

It is expected that the discussion of the present section will also help identify the problems of interest which require experimental investigation in the Shuttle/Spacelab era.

Theoretical models relevant to the interaction of a satellite and a planetary ionosphere

As seen from Tables 1 and 2 the terrestrial and the planetary ionosphere is a highly rarefied collisionless plasma. Therefore, the steady state flow of such a plasma around a satellite is described by the self-consistent solution of the Vlassov-Poisson equations given in the form:

$$
\begin{gathered}
\frac{\partial f_{+}}{\partial t}+\underline{V} \cdot \frac{\partial f_{t}}{\partial \underline{r}}+\frac{e}{M_{+}}\left[\underline{E}+\frac{1}{c}\left(\underline{V}+\underline{V}_{s}\right) \times \underline{B}_{E}\right] \cdot \frac{\partial f_{+}}{\partial \underline{V}}=0 \\
\frac{\partial f_{e}}{\partial t}+\underline{V} \cdot \frac{\partial f_{e}}{\partial \underline{r}}-\frac{e}{M_{e}}\left[\underline{E}+\frac{1}{c}\left(\underline{V}+\underline{V}_{s}\right) \times \underline{B}_{E}\right] \cdot \frac{\partial f_{e}}{\partial \underline{V}}=0 \\
\nabla^{2} \phi=-4 \pi e\left|\int f_{+} \mathrm{d} V-\int f_{e} \mathrm{~d} V\right| \\
=-4 \pi e\left|N_{+}-N_{e}\right| \\
\underline{E}=-\operatorname{grad} \phi
\end{gathered}
$$

where $f_{+}(t, \underline{r}, \underline{v})$ and $f_{e}(t, \underline{r}, \underline{v})$ are the distribution functions of the charged particles (ions and electrons), $N_{+}$and $N_{e}$ their densities and $B_{E}$ is the magnetic field of the Earth. The electric field $E$ is caused by both the potential field of spacecraft and the space charge.

The boundary conditions for the potential $\phi$, are: $\phi=\phi_{B}$ ar $r=R_{0}$ and $\phi=0$ for $r \rightarrow \infty$. The value of $\phi_{B}$ is determined from the condition that the total current to the spacecraft equals zero. The currents include the collection of positive ions and electrons as well as contributions from photoemission, magnetic field effects, r.f. radiation, secondary emission, etc. (Kasha, 1969). Since the distribution of charged particles and the electric potential are interdependent, this system of equations must be solved in a self-consistent manner. 
An expression often used fo, $\phi_{B}$ not obtained with a self-consistent approach) is:

$$
\phi_{B}=-\frac{K T_{e}}{e} \ln \left\{\frac{2\left|\frac{K T_{e}}{2 \pi M_{e}}\right|^{1 / 2}}{\left|\frac{8 K T_{+}}{\mu M_{+}}+V_{s}^{2}\right|^{1 / 2}\left|1-\frac{e \phi_{B}}{E_{+}}+\frac{i_{p h}}{n e}\right|}\right\}
$$

where $E_{+}$is the energy and $i_{p h}$ is the photoemission current density.

In obtaining expression (4) consideration was given to the ion and electron current collection (using simplified expressions) and to the photoelectric current when $i_{p h}$ depends on the incident solar flux, the surface material and the state of cleanliness of the surface (Whipple, 1965; Samir and Willmore, 1966; Fahleson, 1967). No rigorous consideration was given in this expression to magnetic field effects, wake effects, secondary emissions, etc. It should be noticed that the condition of total current to the spacecraft equals zero is correct only if we consider the surface of the spacecraft to be a perfect conductor. If the surface is of insulating materials then a more restricted condition must be used, i.e. the net current is zero at each point on the surface.

In most theoretical studies presently available eqn (2) has been replaced by an assumed electron density of the form $N_{e}(r)=N_{e o} \exp \left(e \phi / k T_{e o}\right)$, where $N_{e o}$ is the ambient electron density and $T_{e o}$ the ambient electron temperature. The method of calculating $N_{+}$is still a subject of controversy. Different approaches and assumptions are used by various researchers.

One approach (Gurevich, 1962, 1970; Al'pert 1976) is to consider the ions as neutrals, thereby eliminating the influence of the potential field due to both the spacecraft potential and the space charge on ion trajectories. In some treatments, the influence of a given potential distribution on the ion trajectories was taken into consideration, but the solution was not self-consistent.

Differences between results of theoretical studies are mainly due to the following factors:

(i) Assumptions concerning the spacecraft equilibrium potential or spacecraft charging.

(ii) Assumptions concerning surface properties of the body-most theories assume a perfect conductor and charge neutralization at the surface. More exact approaches consider various possible charge accommodation-re-emission models for the surface and the possibility of conduction, ranging from perfect to insulating.

(iii) The distribution function used for ions-many treatments assume the ions to be cold (no thermal motion). If thermal motion is included, it may be considered Maxellian, or more properly in some regions, non-Maxwellian.

(iv) The distribution function used for electrons-most treatments assume a Maxwell-Boltzman distribution, however, some non-equilibrium may exist near the body.

(v) The above assumptions apply primarily to kinetic theories. However, some early treatments used a continuum-gas-dynamic approach where no charge separation was allowed. 
(vi) The use of various numerical integration techniques.

It is clear that understanding the physical mechanisms responsible for creating the wake and the disturbed patterns elsewhere around the spacecraft, depend partially on the factors mentioned above. Moreover, most studies assume $B_{E}=0$, and limit therselves to solving eqns (1)-(3) for steady state conditions, i.e. $(\partial f / \partial t)=0$.

It should be realized that fundamental questions such as the existence (or non-existence) of shock patterns behind the satellite have not been examined critically. Neither is it clear at present whether oscillations exist in the region of disturbed plasma flow around the satellite. However, there are good reasons to believe that this is indeed the case (Samir and Willmore, 1965 for experimental work; Gurevich et al., 1970; Al'pert, 1976 for theoretical considerations).

As far as the plasma flow regimes are concerned, most of the relevant theoretical models assume the plasma to be collisionless, i.e. the electron-ion mean free path to be much larger than the linear dimensions of the satellite, (see Table 3). It is also commonly assumed that the satellite velocity is larger than the ion thermal velocity and much smaller than the electron thermal velocity, which is generally valid for satellites below $10^{4} \mathrm{~km}$ altitude. On the other hand not all models consider realistic values for $R_{D}=\left|R_{0} / \lambda_{D}\right|$, which for the practical case of an ionospheric satellite, $R_{D} \gg 10$.

The problem of calculating the charged particle density behind spacecraft having the same cross-sectional area but different shapes, was discussed theoretically by Al'pert et al. (1965), Maslennikov and Sigov (1968) and Al'pert (1976). The influence of the shape of the body is critical for the region close to the spacecraft surface. The subject of the influence of the satellite shape, crosssectional area and the dimension of the body in the stream direction was discussed explicitly by Gurevich et al. (1970). The studies by Al'pert et al. (1965) and by Gurevich et al. (1970) do not contain a detailed trajectory calculation for obtaining the ion density around the body and use simplified assumptions. Therefore their degree of applicability to the question of the reliability and quality of in situ measurements is limited.

Parker (1976) developed a computer program which can be employed for studies of some of the disturbed zones around a spacecraft under specific and fairly applicable ionospheric conditions. The program solves the coupled system of Vlassov-Poisson eqns (1)-(3) in a self-consistent manner to obtain ion and electron densities and the potential about three-dimensional bodies with axial symmetry about the direction of the plasma flow. This program retraces ion and electron trajectories from the point in space at which it is desired to know the velocity distribution to their origin in the undisturbed plasma where the distribution is known. This method is referred to as the "inside-out" method (see Parker, 1977; Whipple, 1977) as compared with the "outside-in" method which follows particle trajectories in the particles natural direction of motion.

Parker's approach differs from earlier studies (Call, 1969; Gurevich et al., 1970; and others) in that it includes both the ion and electron thermal motions and bodies (representing the spacecraft), are not considered infinite in their dimensions. Further, the Parker model, unlike say, the Grabowski and Fisher (1975) model, does not assume that quasi-neutrality holds throughout. A more 
complete discussion of the advantages of the Parker theory over other theoretical models is given in Whipple (1977). It is not yet clear, however, over what range of plasma parameters the Parker theory holds. In particular, it is not clear if this theory applies to large bodies (i.e. large values of $R_{0} / \lambda_{D}$ ) and to a variety of spacecraft geometries. The case of the Shuttle/Spacelab, which may be subject to various phenomena of differential charging, will very definitely require special treatment not yet provided by any single theoretical model.

Mathematical details of Parker's sheath and wake model for a pillbox-shaped satellite including some preliminary sample results applied to in situ data from the Ariel I and Explorer 31 satellites (Henderson and Samir, 1967; Samir and Jew, 1972), are given elsewhere (Parker, 1977). Goddard (1975) carried out a detailed approximate calculation of the interaction of spherical and cylindrical collectors, e.g. satellites and/or plasma probes with a collisionless Maxwellian plasma. Although the study treats the case $(\partial f / \partial t)=0, B_{E}=0$ and assumes the cylinders to be infinite, its comparison with other, more exact theories (Fournier, 1971) which employ a self-consistent approach, is very useful. The study was performed in a parametric manner, i.e. computing the flow field contours as a function of the parameters $R_{D}, S, \phi_{N}$ and $T\left[=\left(T_{e} / T_{+}\right)\right]$. Goddard claims agreement with Fournier to within $5 \%$ for specific cases. The latter study should be continued and extended to include other theoretical models. It would be of great value if reasonable and proper practical assumptions could be made that could replace the complicated self-consistent calculations. The latter are very expensive and often prohibitive financially.

As indicated above, there are quite a number of models which claim real applicability to the practical case of the interaction between satellite and the terrestrial ionosphere. However, very often the models vary significantly and, with some exceptions, such as Goddard (1975), there are few intertheory comparisons. Further, as a result of the assumption used, there is no meaningful way to check the usefulness of the models to real cases in space. A few attempts were made in the past ten years $\dagger$ to compare results from in situ observations with theoretical sheath/wake models, but at the present time the above effort should be viewed as "exploratory".

As mentioned earlier in this section, most theoretical investigations of the interaction of a flowing plasma with a body have treated only steady-state conditions, assuming that no time dependent process exist, i.e. $\partial / \partial t=0$. However, even under steady-state conditions (i.e. after the characteristics of the ambient plasma have remained constant for a long time so that the disturbance around the body has reached its"steady-state" condition) there remains the possibility of the continuous generation of plasma instabilities and oscillations.

Gurevich et al. (1970) studied plasma stability in the wake of a body moving supersonicly through a highly rarefied plasma medium. They show that secondary plasma fluxes (or streams) move through each other a short distance downstream from the body.

†See Liu, 1969; Gurevich et al., 1970; Samir, 1973; Samir and Jew, 1972; Samir et al., 1975; Parker, 1977; and Al'pert, 1976. 
The effective temperature of the ions $T_{+}(\mathrm{eff})$ in these two fluxes is exponentially small while the temperature of the electrons is the same as in the ambient plasma. Consequently, $T_{e} \gg T_{+}$(eff). Therefore, it could be expected that in this region some instability analogous to a two-stream instability in a plasma with different temperatures of ions and electrons may appear. Such an instability may lead to the generation of plasma oscillations with frequencies which, for a typical ionospheric particle density of $10^{5}$ and ionic mass $M_{+}=16$, is $\simeq 5 \mathrm{kc} / \mathrm{sec}$. This will be shown in the following section to be in accord with experimental predictions based on data from the Ariel I satellite.

The question of the excitation of waves and the instability of the plasma around a rapidly moving body was discussed recently in great detail by Al'pert (1976) and by Liu (1975). Al'pert (1976) discussed the instability conditions of the plasma in the vicinity of the body, the types of oscillations excited in the disturbed region of the plasma and carried along with it, and the possibility of emission of electromagnetic oscillations from the disturbed region of the plasma. He suggests that the character of the disturbances in the wake, considering electric and magnetic effects, is associated with the excitation of ion-accoustic waves.

It should be noted that the time dependent treatments discussed here are not theoretical models of the entire interaction but more in the nature of an investigation of specific physical processes. In this same spirit, time independent but nonlinear processes in rarefied plasma dynamics have been studied by Gurevich and Piraevsky (1975). Recently, Kunemann (1978) computed the ion density in the wake of bodies moving in collisionless plasma flows. The approach is essentially qualitative and yields differences between the flow patterns around point-like bodies and bodies with prescribed finite dimensions. A great part of the discussion is devoted to plasmas with $T_{e} \gg T_{+}$. In discussing the results of wake features, the semi-quantitative computations were compared with laboratory simulation studies. For application to the case of large bodies $\left(R_{D} \gg 1\right)$ and for isothermal plasmas $\left(T_{e} \approx T_{+}\right)$the prediction is that the wake will be quite similar to the neutral particle approximation at least if the surface potential is not too large (i.e. $\phi_{N}$ relatively small).

Isensee (1977) assessed the plasma disturbances caused by the Helios spacecraft in the solar-wind. The situation here is different from the case of the interaction between a satellite and the ionosphere since the characteristics of the plasma flows are different, e.g. the significance of the photocurrents. Moreover, at high altitudes the contribution of high energy particles to the content of the plasma is great but on the other hand, the disturbances created to that component of the plasma is less significant than the disturbances on the "low energy" component of the plasma (the latter being the main component for planetary ionospheres).

Significant results from in situ investigation-a comparative examination

Most of the information available from in situ measurements which is relevant to satellite-ionospheric interactions comes from (1) The Ariel I Satellite (Samir and Willmore, 1965, 1966; Henderson and Samir, 1967), (2) from the Ex- 


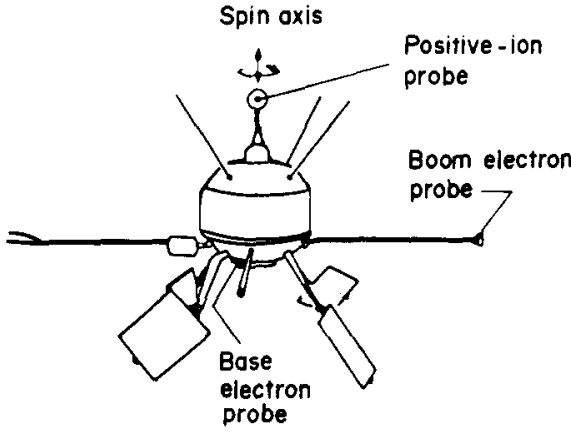

(a) Ariel I

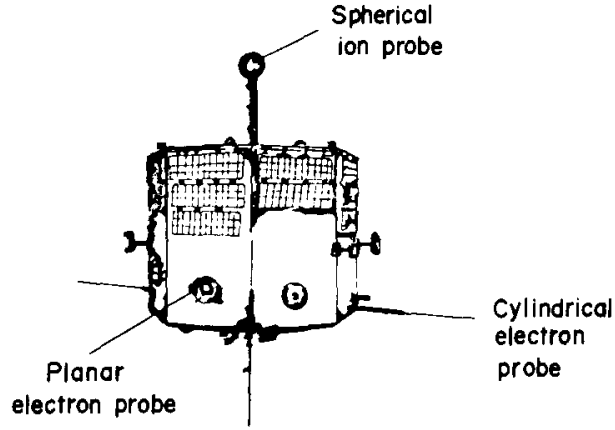

(b) Explorer

Fig. 2. Schematic drawing showing the ionospheric satellites (including probe location) from which measurements were used for the study of satellite-ionosphere interactions.

lorer 31 Satellite (Samir and Wrenn, 1969, 1972; Samir et al., 1973, 1975; Troy et al., 1975) and (3) from the Atmosphere Explorer C Satellite (Samir et al., 1979a; Samir et al., 1979b. The geometry and instrument locations for the Ariel I and Explorer satellites are shown in Fig. 2. The Ariel I information was exploratory in nature and showed the existence of a wake zone behind the satellite which is depleted of both ions and electrons with a net negative space charge. The results from the Explorer 31 enhanced our knowledge in a more quantitative manner.

Most of the available results prior to 1973 (Samir, 1973a, b) gave the angular distribution of electron current (and/or density) at the closest vicinity to the satellite's surface for several altitudes, i.e. $I_{e}\left(N_{e}\right)=f(\theta)$ at $r=R_{0}$ where $I_{e}$ and $N_{e}$ are the electron current and density respectively, $\theta=$ angle between the satellite velocity vector and the normal to the measuring probe, $r=$ radial distance from the center of the satellite and $R_{0}=$ effective radius of the satellite. Figure 3 shows a typical example of $I_{e}=f(\theta)$ behavior for the altitude ranges $500-600 \mathrm{~km}$ and for $2900-3000 \mathrm{~km}$. This figure depicts an average behavior using measurements from a large sample of satellite passes. These observations were obtained from probes which were flush-mounted on the satellite surface. Figure 4 (after Samir and Wrenn, 1969) provides some more detailed examples of the variation of $I_{e}=f(\theta)$ for the altitude ranges: (a) $532-660 \mathrm{~km}$ (b) $620-910 \mathrm{~km}$, (c) $1175-1685 \mathrm{~km}$ and (d) 2060-2280 km. Very little was known prior to 1973 about the angular distribution of ions around an ionospheric satellite. In order to contribute to the question of charging mechanisms, and to the effect of satellite potential on electric drag, the potential of the Ariel I satellite when in the shadow of the Earth was compared with its potential when in sunlight. No significant effect was observed. Moreover, the approximate value of the photoemission current density was assessed (Samir and Willmore, 1966), and the value is still in accord with the present day values obtained by more sophisticated means.

The main deficiency of the early studies is that no systematic parametric investigation was performed. This is so, since the needed ensemble of plasma 


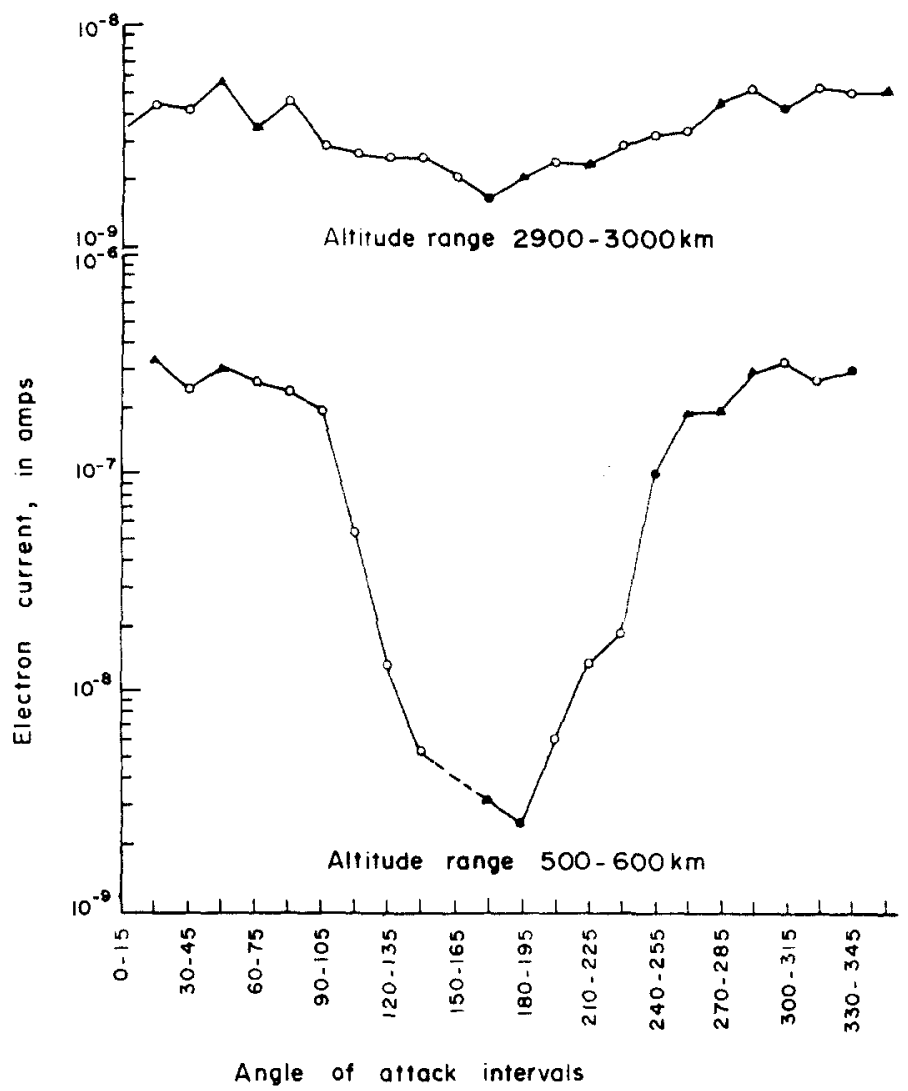

Fig. 3. Average values for the variation of $I_{e}=f(\theta)$ for the altitude ranges: $500-600 \mathrm{~km}$ and 2900-3000 km. Explorer 31 data (After: Samir 1973b).

parameters was not available due to the lack of relevant and/or reliable in situ measurements. However, despite the very meagre amount of information, an attempt was made to compare the wake of the main body of the Ariel I satellite with the wake created by the spherical ion probe (Henderson and Samir, 1967). The idea being to compare the wake behind bodies of different potential and different size at different distances downstream of the wake-creating body. Recently Parker compared results from his elaborate wake model with these experimental results, (Parker, 1977). At the present time, however, the status of such theory-experiment comparison should be considered preliminary and more work in this direction is in progress.

In studying the measurements from the Explorer 31 satellite, the scientific objective was to extend the results of the Ariel I satellite to a wider range of altitudes hence to a wider range of plasma parameters including the examination of the angular distribution of the local potential field around the satellite (Samir and Wrenn, 1969). The apogee of the Explorer 31 satellite was about $3000 \mathrm{~km}$ whereas that of the Ariel I satellite was about $1200 \mathrm{~km}$. The result for $I_{e}=f(\theta)$ 


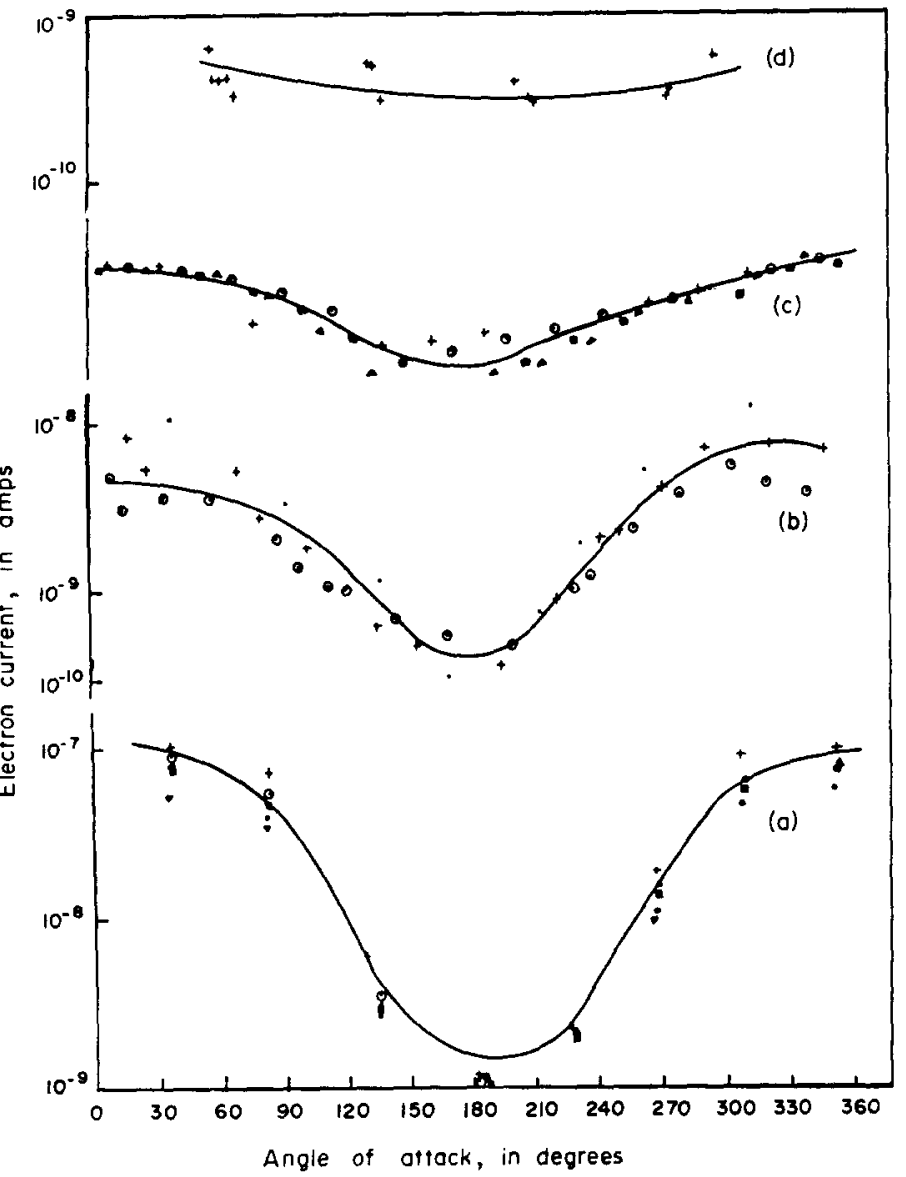

Fig. 4. Variation of $I_{e}=f(\theta)$ in the altitude ranges: (a) $432-600 \mathrm{~km}$, (b) $620-910 \mathrm{~km}$, (c) $1175-1685 \mathrm{~km}$, and (d) $2060-2280 \mathrm{~km}$ (After: Samir and Wrenn, 1969).

shown in Fig. 3 for the altitude range $500-600 \mathrm{~km}$ are in agreement with the main result from the Ariel I satellite namely, that:

$$
\left|\frac{\left.I_{e} \text { (wake) }\right)}{\left.I_{e} \text { (ambient }\right)}\right|_{r=R_{0}} \approx\left|\frac{\left.I_{e} \text { (wake }\right)}{\left.I_{e} \text { (front) }\right)}\right|_{r=R_{0}} \simeq 10^{-2}
$$

for the altitude range $500-700 \mathrm{~km}$ where $\left|\mathrm{O}^{+}\right|$is the major ionic constituent. Here $I_{e}$ (wake) $\equiv I_{e}$ (at $\theta=180^{\circ} \pm 15^{\circ}$ ) and $I_{e}$ (front) $\equiv I_{e}$ (at $\theta=0^{\circ} \pm 15^{\circ}$ ). Using a relatively small sample of measurements from the Ariel I satellite it was found that there may be a possible enhancement in ion current at the "inner boundary" of the wake zone. The Ariel I satellite also had a planar, circular guarded probe, similar to the surface mounted probes on Ariel I and the Explorer 31 satellites, which was mounted on a boom that extended 5 radii from the center of the 
satellite. Hence, it was possible to examine $I_{e}=f(\theta)$ at $r=5 R_{0}$. It was found that

$$
\left|\frac{N e \text { (wake) }}{N e \text { (ambient })}\right|_{r=5 R_{0}} \approx 0.5 \pm 0.1
$$

for a plasma with $S\left[\equiv\left(V s / V_{T}(+)\right)\right]=3.75$ and $R_{D}[\equiv(R o / \lambda D)] \approx 10$. A comparison of the latter result with the result at $r=R_{0}$ allowed a crude assessment of the gradient of $I_{e}$ across a distance of $r=4 R_{0}$ in the wake. Needless to say, more work is needed to substantiate $\left|\left(\Delta I_{e} / \Delta r\right)\right|$ gradient vs a wider range of plasma parameters and properties. Results of the first attempt to investigate the variation of $\mid\left(I_{e}\right.$ (wake) $/ I_{e}$ (ambient)) $\mid=F\left(\left|M_{+}\right|_{A V}\right)$ is shown in Fig. 5. This was the first effort to investigate the dependence of electron current on plasma parameters using in situ observations. Since the range of variability of $\phi_{N}$ and $R_{D}$ for the above sample is not known the result given in Fig. 5 should not be considered a "pure" $I_{e}=f\left(M_{+}\right)$dependence. Analysis of measurements from the cylindrical probes on the Explorer 31 (Brace and Findlay, 1969 and Findlay and Brace, 1969) yielded the $I_{e}$ and $N_{e}$ across a cylindrical collector, which spans over a range of about $1 R_{0}$ at a distance of about $2 R_{0}$ from the satellite's center. Therefore,

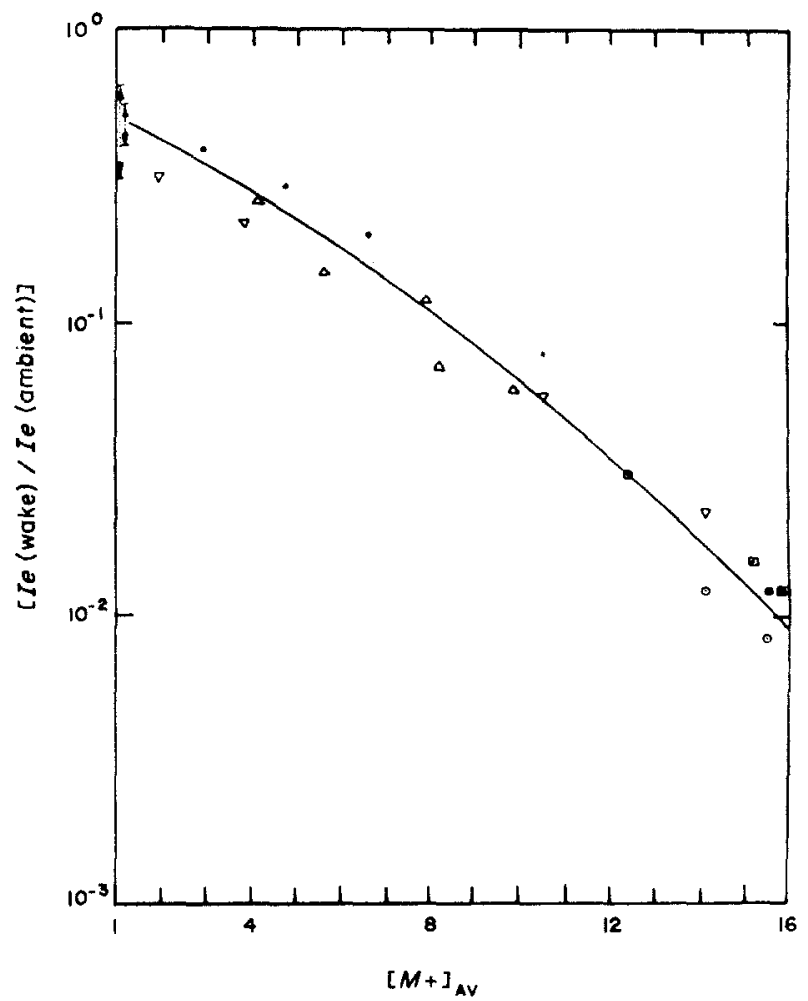

Fig. 5. Variation of $\mid\left[I_{c}\right.$ (wake) $/ I_{e}($ ambient $\left.)\right] \mid=f\left(\left|M_{+}\right|_{A V}\right)$ from Explorer 31 measurements in the altitude range $520-1020 \mathrm{~km}$, (After: Samir, 1973a). 
rather than giving $N_{e}(\theta)$ at a specific fixed distance, $r$, from the center of the satellite (as was the case for the Ariel $I$ and Explorer 31 results discussed above), the cylindrical probe gives $N_{e}(\theta)$ averaged over a range $\Delta r=R_{0}$ located at a fixed distance. The results from the above investigation show:

$$
\left|\frac{N e\left(\theta \approx 180^{\circ}\right)}{N e\left(\theta=0^{\circ}\right)}\right|=\left|\frac{N e \text { (wake })}{N e(\text { ambient })}\right| \approx 3-5
$$

in the altitude range $520-711 \mathrm{~km}$. Miller (1972) analyzed a large sample of cylindrical probe measurements from the Explorer 31 and found an electron current enhancement near $\theta \sim 120^{\circ}$. We should call attention to the fact that a similar $N_{+}$enhancement was implied by the Ariel I results mentioned earlier (Samir and Willmore, 1965). Al'pert et al. (1965) and Al'pert (1976) discuss the possibility of the existence of such an enhancement and relate it to electric field effects. It is our impression that these findings require further experimental substantiation before they can be considered a real physical effect.

Using Ariel I measurements, the existence of ion plasma oscillations located in the boundary region of the wake was predicted (Samir and Willmore, 1965). The frequency of the predicted ion plasma oscillations was found to have a frequency component of about $3 \mathrm{kc} / \mathrm{sec}$. This is in close agreement with theoretical predictions by Al'pert (1976) based on a "two-stream" type instability, as discussed in the previous section.

The angular distribution of electron temperature measured by the a.c. planar guarded probes mounted on the surface of the Explorer 31 satellite was also investigated. It was found that situations exist where the electron temperature in the very near wake exceeds that of the ambient plasma. Figure 6 shows an example of the variation of normalized electron temperature $\mid T_{\epsilon}$ (normalized) $\mid=$ $\mid\left(T_{e}(\theta) / T_{e}\right.$ (front))| indicating $\left|T_{e}(\theta)\right|$ to be enhanced by $50 \%$ in the $\theta=$ $180^{\circ}$ position. The results of Fig. 6 are for the altitude $800-1000 \mathrm{~km}$ where the dominant ion was $\mathrm{O}^{+}$and the satellite velocity $\simeq 8 \mathrm{~km} / \mathrm{sec}$.

Another example showing $\mid\left(T_{e}\right.$ (wake) $\mid / T_{e}$ (ambient) $) \mid>1$ is shown in Fig. 7. It is seen that the electron temperatures for $\theta=180^{\circ} \pm 15^{\circ}$ or $\theta=180 \pm 30$ are consistently higher than those for: $\theta=0+15^{\circ}$ or: $\theta=0 \pm 30^{\circ}$. The magnitude of the temperature enhancement is probably plasma parameter dependent but no systematic parametric study has yet been performed to clarify the above dependence.

It should be noted that $\mid T_{e}$ (wake) $\mid$ measurements from the cylindrical electrostatic probes on board the Explorer 31 (representing the situation at $r \approx 2 R_{0}$ from the surface of the satellite) did not show any similar enhancement in $\mid T_{e}$ (wake)|.

Henderson and Samir (1967) reported that they could not detect any deviations from the ambient electron temperature in the wake of the Ariel I satellite at a distance of $5 R_{0}$ downstream from the center of the satellite. This implies that the $\mid T_{e}$ (wake) $\mid$ enhancement is confined to the near vicinity of the surface. The cause and nature of this $\mid T_{e}$ (wake) $\mid$ enhancement is not yet clear. However, since 


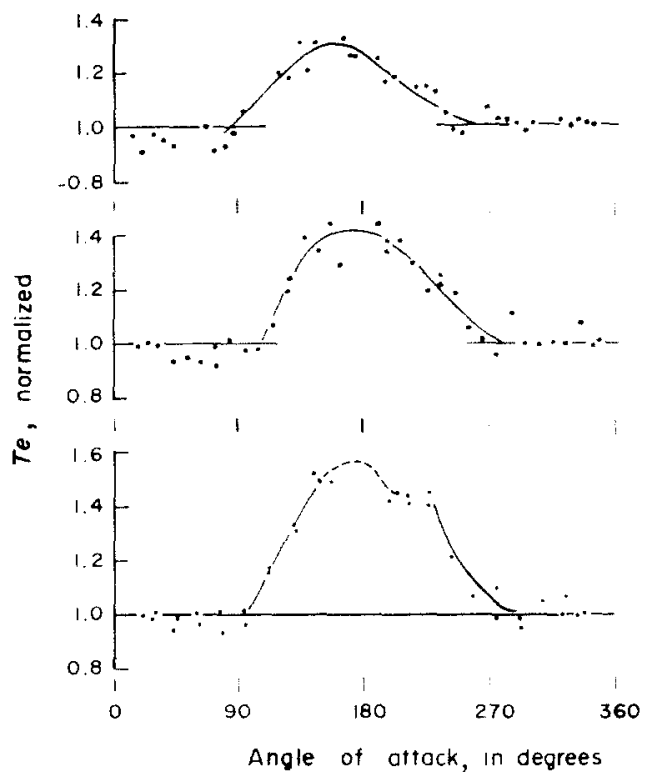

Fig. 6. Variation of normalized electron temperature with angle of attack, in the altitude range $800-1000 \mathrm{~km}$, dominant ion $=\mathrm{O}^{+}$(After: Samir and Wrenn, 1972).

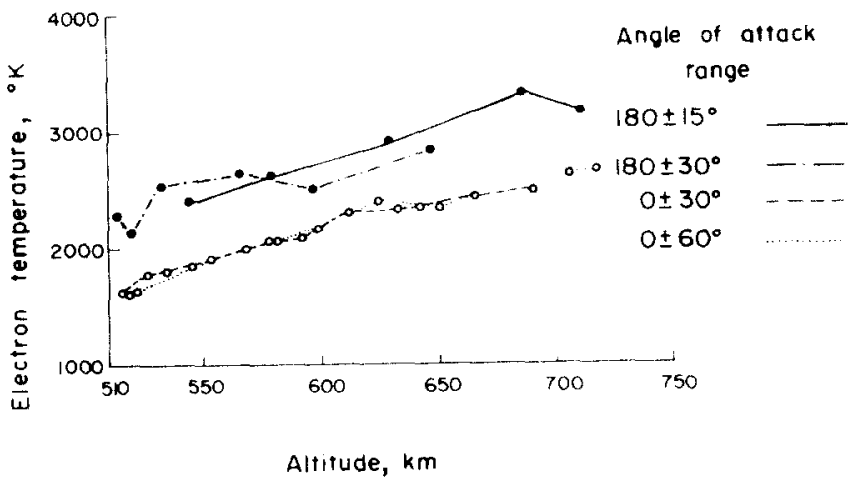

Fig. 7. Variation of electron temperature with altitude where: $0^{+}$is the major ionic constituent. Explorer 31 data (After: Samir and Wrenn, 1972).

a negative potential well is believed to exist behind the satellite $\dagger$ it is possible that in this well, wave-particle interactions take place which energize the electrons that leave the well. The resulting population of electrons close to the satellite can then have an effective temperature which is higher than that of the ambient thermal electrons. Alternatively, one might infer the existence of heating mechanisms in the wake region due to stream interactions

†See Liu, 1969; Gurevich et al., 1970; Al'pert, 1976; Isensee, 1977; and Kunemann, 1978. 
and/or instabilities correlated with plasma oscillations in the near wake (Al'pert 1976).

These possibilities remain as yet speculative until more theoretical and experimental work can be done. In particular, a measurement of the complete energy distribution of the wake population would clarify the situation and establish the true significance of the "temperature" determinations. The question of the $\mid T_{e}$ (wake)| enhancement has also been investigated by Illiano and Storey (1974) in the laboratory and by Troy et al. (1975) using more in situ data from the Explorer 31 satellite. The conclusions of the studies are not in accord.

Since 1973 the main effort which utilizes in situ data concentrates on a detailed parametric investigation aimed at studying the dependence of the ion population in the near vicinity of the satellite. Ion measurements made on the Explorer 31 by a retarding potential analyzer (Donley, 1969; Maier, 1969) were used in order to study the angular distribution of the ion flux in the nearest vicinity to the spacecraft surface. The retarding potential analyzer was mounted on the satellite's equatorial plane with its aperture normal situated perpendicular to the satellite spin axis. Figure 8 shows the variation of the normalized particle fluxes (both ion and electron) with the angle of attack, $(\theta)$. As could be expected $N_{+}(\theta) \ll N_{e}(\theta)$, the inequality being greater as the maximum wake position is approached. The results given in Fig. 8 are for $I_{+}(\theta)$ and $I_{l}(\theta)$ in the altitude

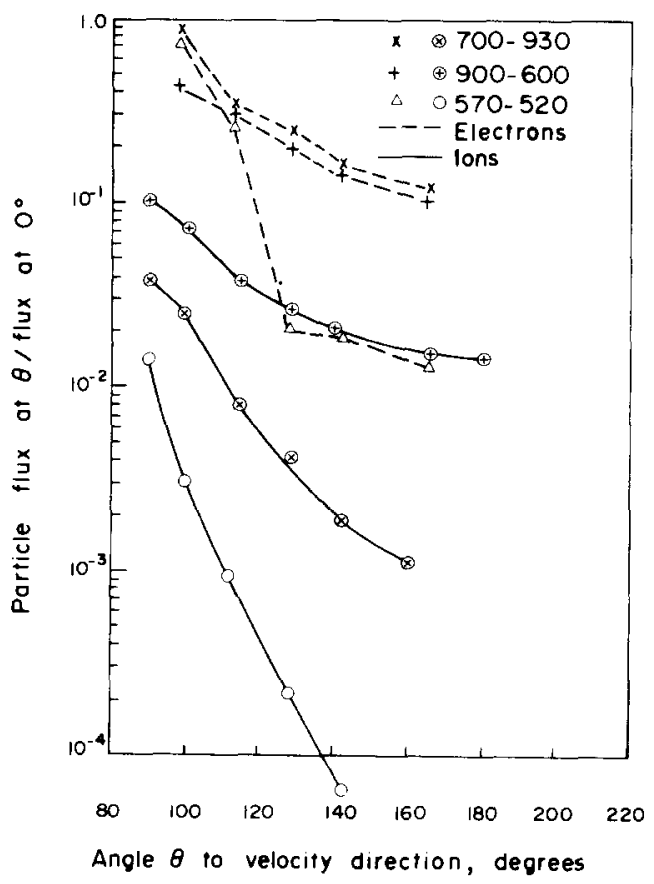

Fig. 8. Variation of normalized ion and electron currents with angle of attack $(\theta)$ in the angular range: $90^{\circ} \leq \theta \leq 180^{\circ}$ and for the altitude ranges: $700-930 \mathrm{~km}, 900-600 \mathrm{~km}$ and $570-520$ km (After: Samir et al., 1973). 
Table 8. Plasma properties and plasma parameters for the results given in Fig. 8 Explorer 31 data (after: Samir et al., 1973)

Ionospheric plasma properties

Plasma parameters

\begin{tabular}{|c|c|c|c|c|c|c|c|c|c|c|c|c|}
\hline$N_{+}$ & $T$ & $T_{i}$ & & & & $\mathrm{H}^{+}$ & 0 & $M$ & & & & \\
\hline$\left(10^{4} / \mathrm{cm}^{3}\right)$ & & $\left({ }^{\circ} \mathbf{K}\right)$ & (Vol & & & Densitie & $\left.0^{3} / \mathrm{cm}^{3}\right)$ & (AM & J) $\frac{R_{0}}{\lambda_{D}}$ & $\frac{T_{0}}{T_{+}}$ & $\overline{M_{+}}$ & $\frac{e \phi_{s}}{k T_{\varepsilon}}$ \\
\hline 0.84 & 1426 & 1406 & -0.82 & 6.9 & 6.9 & 1.5 & 0.1 & 1.6 & 14.1 & 1.01 & 1.8 & -6.7 \\
\hline 0.42 & 1649 & 1730 & -0.79 & 6.2 & 4.0 & 0.2 & 0.0 & 1.1 & 9.3 & 0.95 & 1.2 & -5.6 \\
\hline 2.6 & 1876 & 1631 & -0.75 & 7.9 & 6.8 & & 19.0 & 12.0 & 21.6 & 1.15 & 4.8 & -4.6 \\
\hline 2.1 & 1933 & 1850 & -0.77 & 7.8 & 5.2 & & 15.0 & 12.1 & 19.1 & 1.04 & 4.8 & -4.6 \\
\hline & 2116 & & -0.71 & 7.8 & & & & & & & & \\
\hline & 2236 & & -0.75 & 7.7 & & & & & & & & \\
\hline & 2247 & & -0.72 & 7.7 & & & & & & & & \\
\hline 2.4 & 3117 & 2155 & -0.57 & 7.6 & 10.0 & 6.5 & 6.1 & 5.9 & 16.1 & 1.45 & 2.5 & -2.1 \\
\hline 2.6 & 2906 & 2176 & -0.64 & 7.6 & 11.6 & 2.9 & 10.0 & 7.5 & 17.3 & 1.34 & 3.0 & -2.6 \\
\hline 2.8 & 2897 & 2167 & -0.74 & 7.7 & 10.3 & 3.5 & 14.0 & 8.9 & 18.0 & 1.34 & 3.3 & -3.0 \\
\hline 3.0 & 2914 & 2412 & -0.76 & 7.7 & 9.1 & 2.0 & 18.0 & 10.5 & 18.6 & 1.21 & 3.6 & -3.0 \\
\hline 3.2 & 2989 & 2272 & -0.92 & 7.8 & 8.2 & 2.1 & 22.0 & 11.4 & 19.0 & 1.32 & 3.8 & -3.6 \\
\hline 3.6 & 2962 & 2720 & -0.87 & 7.8 & 5.6 & 2.0 & 30.0 & 13.1 & 19.8 & 1.09 & 4.0 & -3.4 \\
\hline 4.4 & 2933 & 2672 & -0.84 & 7.9 & 5.0 & 0.2 & 38.0 & 14.2 & 22.5 & 1.10 & 4.2 & -3.3 \\
\hline 5.0 & & 2681 & & 7.9 & 3.7 & 0.2 & 45.6 & 14.9 & & & & \\
\hline 2.7 & 2700 & 2698 & -0.73 & 7.7 & 12.0 & & 15.0 & 9.3 & 18.3 & 1.00 & 3.5 & -3.1 \\
\hline 3.0 & 2880 & 2738 & -0.78 & 7.8 & 8.6 & & 21.0 & 11.6 & 18.7 & 1.05 & 3.8 & $-3,1$ \\
\hline 3.3 & 3000 & 2820 & -0.83 & 7.9 & 6.9 & & 26.0 & 12.9 & 20.8 & 1.06 & 4.0 & -3.2 \\
\hline 3.3 & 3250 & 3005 & -0.90 & 7.9 & 4.3 & & 29.0 & 14.1 & 18.5 & 1.08 & 4.0 & -3.2 \\
\hline 3.4 & 3430 & 3029 & -0.95 & 8.0 & 2.1 & & 32.0 & 15.1 & 18.2 & 1.13 & 4.1 & -3.2 \\
\hline 52.0 & 1337 & 1343 & -0.44 & 8.1 & & & 520.0 & 16.0 & 114.3 & 1.00 & 6.8 & -3.8 \\
\hline 33.0 & 1511 & 1424 & -0.53 & 8.1 & & & 330.0 & 16.0 & 85.7 & 1.06 & 6.4 & $\ldots 4.1$ \\
\hline 22.0 & 1742 & 1392 & -0.57 & 8.1 & & & 220.0 & 16.0 & 65.1 & 1.25 & 6.0 & -3.8 \\
\hline 14.0 & 1967 & 1558 & -0.58 & 8.1 & & & 140.0 & 16.0 & 48.9 & 1.26 & 5.6 & -3.4 \\
\hline 13.0 & 2234 & 1579 & -0.66 & 8.1 & & & 130.0 & 16.0 & 44.2 & 1.41 & 5.3 & -3.4 \\
\hline 10.0 & 2660 & 1550 & -0.70 & 8.1 & & & 100.0 & 16.0 & 35.2 & 1.72 & 4.8 & -3.1 \\
\hline
\end{tabular}

ranges; $700-930 \mathrm{~km}, 900-600 \mathrm{~km}$ and $570-520 \mathrm{~km}$. Table 8 gives the plasma parameters for measurements of $I_{+}(\theta)$ over the range $90^{\circ} \leq \theta \leq 165^{\circ}$ (Samir et al., 1973). This information was used for some qualitative theory-experiment comparisons (Samir et al., 1975).

Recently, measurements of ion current electron temperature and values of space potential obtained from the cylindrical electrostatic probe (CEP) on board the Atmosphere Explorer $\mathrm{C}(\mathrm{AE}-\mathrm{C})$ satellite were used to examine in a parametric manner, the angular distribution of $I_{+}(\theta)$. The investigation focused on the angular dependence of $I_{+}(\theta)$ for large values of $R_{D}$ including $R_{D}>10^{2}$, 
Table 9. Some relevant plasma properties and parameters for each of the cases used

\begin{tabular}{crrrrrrr}
\hline & \multicolumn{3}{c}{ Plasma properties $\dagger$} & \multicolumn{3}{c}{ Plasma parameters } \\
Alt. range $(\mathrm{km})$ & $T_{e}(\mathrm{~K})$ & $N_{i}\left(\mathrm{~cm}^{-3}\right)$ & \multicolumn{1}{c}{$\phi_{s}(\mathrm{~V})$} & $\left|R_{0} / \lambda_{D}\right|$ & $\left|T_{e} / T_{i}\right| \ddagger$ & $S_{(A V) \S}\left|e \phi_{s} / K T_{e}\right|$ \\
\hline $276-313$ & 970 & $2.65 \times 10^{5}$ & -0.802 & 135.7 & 1.08 & 8.04 & 9.4 \\
$360-404$ & 977 & $1.93 \times 10^{5}$ & -0.816 & 116.3 & 1.09 & 7.97 & 9.5 \\
$450-508$ & 948 & $9.87 \times 10^{4}$ & -0.770 & 83.8 & 1.07 & 7.94 & 9.3 \\
$558-600$ & 991 & $4.98 \times 10^{4}$ & -0.755 & 58.0 & 1.21 & 5.93 & 8.7 \\
$357-411$ & 1039 & $4.02 \times 10^{5}$ & -0.771 & 162.5 & 1.11 & 7.70 & 8.4 \\
$475-538$ & 948 & $7.48 \times 10^{4}$ & -0.732 & 73.4 & 1.14 & 7.83 & 8.8 \\
\hline
\end{tabular}

$\dagger T_{e} I_{+} \phi_{s}$ from CEP measurements.

$\ddagger T_{i}$ from RPA measurements.

§Ion composition from BIMS measurements.

which is of practical use to Shuttle/Spacelab and large space station experiments.

Table 9 presents some relevant plasma properties and parameters used in this investigation. Due to the wide complement of instruments on board the Atmosphere-Explorer satellites it was not too difficult to compute the parameters given in the table from the actual measurements (Samir et al., 1979a, b).

The results for the $I_{+}(\theta)$ distribution around the AE-C satellite are for $r \sim 2.5$ $R_{0}$ from the center of the satellite and yield an (empirical) exponential relationship for $\mid\left[I_{+}(\theta \sim 160) / I_{+}(\right.$ambient $\left.)\right] \mid=f\left(R_{D}\right)$. This is shown in Fig. 9(a). More details on the above study are given elsewhere (Samir et al., 1979a). The variation of $\left[i_{+}\left(\theta \sim 160^{\circ}\right) / I_{+}(\right.$ambient $\left.)\right]=f\left(S_{A V}\right)$ shows an approximate linear dependence (see Fig. 9b).

Since the above results are based on relatively small samples of measurements, it would be very useful to test the existence of the above relationships for a wider range of plasma parameters.

Recently (Samir et al., 1979b), a study was performed which examined the influence of electron temperature and relative ionic composition on the ion depletion in the wake of the AE-C satellite. It was observed that both electron temperature and ionic composition significantly influence the amount of ion depletion in the near wake zone. For example it was found that the ion current in an $\mathrm{O}^{+}$-dominated plasma decreases (with respect to ambient) by about two orders of magnitude at $T_{e} \sim 10^{3} \mathrm{~K}$ and by a factor of about 30 at $T_{e} \sim 3 \times 10^{3} \mathrm{~K}$. Whereas for a plasma with $\left|\left[N\left(\mathrm{O}^{+}\right) / N\left(\mathrm{H}^{+}\right)\right]\right| \approx 1$ (where: $N\left(\mathrm{O}^{+}\right)$and $N\left(\mathrm{H}^{+}\right)$ represent $\mathrm{O}^{+}$and $\mathrm{H}^{+}$density respectively) the ion current decreases by a factor of 6 (in the wake) at $T_{e} \sim 10^{3} \mathrm{~K}$ and by a factor of 2.3 at $T_{e} \sim 3 \times 10^{3} \mathrm{~K}$. The main findings of the above investigation are shown in Fig. $9(\mathrm{c})$ where the quantitative dependence of $\alpha=\mid\left[I_{+}\right.$(wake) $/ I_{+}$(ambient) $] \mid=f\left(T_{e}\right)$ for constant values of the ratio $R=\left[N\left(\mathrm{O}^{+}\right) / N\left(\mathrm{H}^{+}\right)\right]$is given. As seen from this figure, the ratio $\alpha=$ $f\left(T_{e}, R\right)$. Figure $9(\mathrm{c})$ confirms the anticipated result (Al'pert, 1976; Kunemann, $1978)$ that $(\alpha)$ should increase with decreasing values of $R$. 


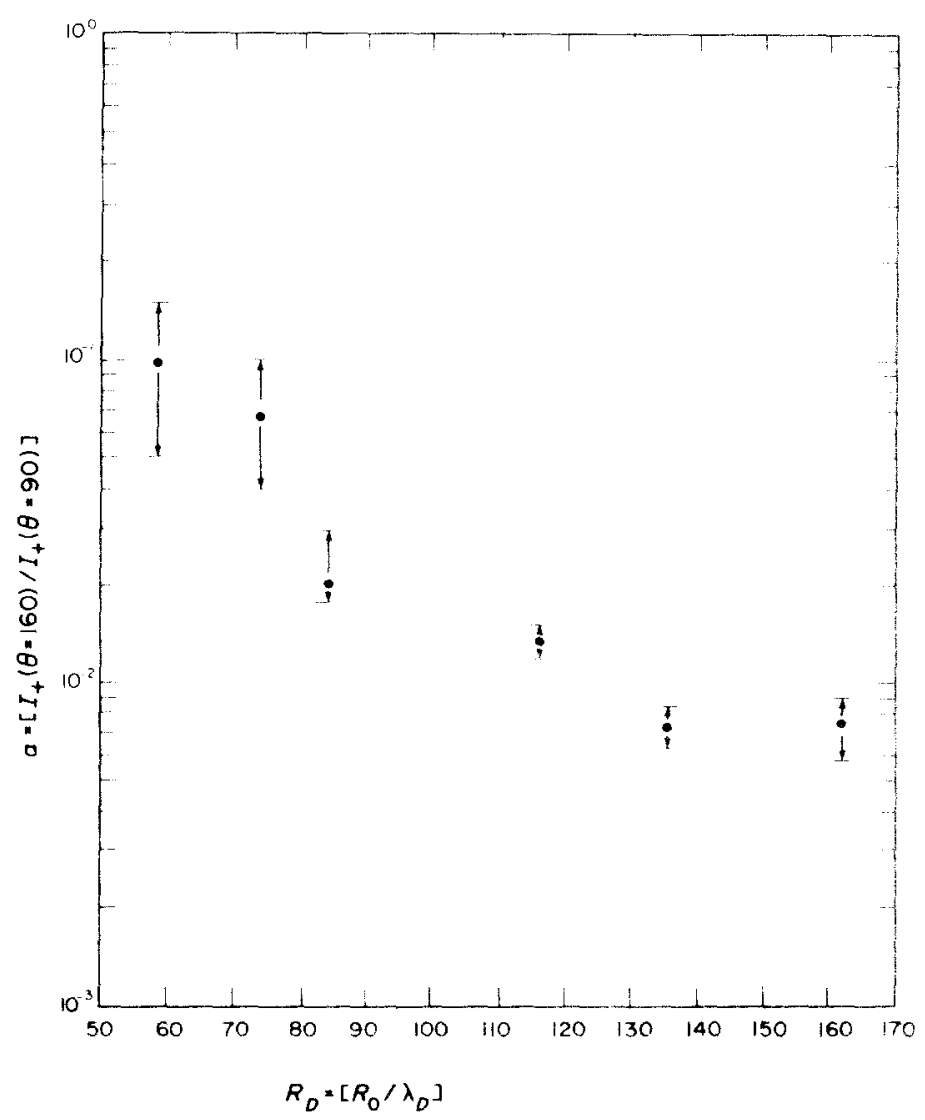

Fig. 9(a). Variation of $a=\left[\ell_{+}(\theta=160) / L_{+}(\theta=90)\right]$ with $R_{D}\left(=R_{0} / \lambda_{D}\right)$

Controlled in situ experiments using the Shuttle/Spacelab system for the in vestigation of rarefied plasma flow interactions with objects in space

\section{General}

In this section we discuss, in some detail, how future experiments yielding a high scientific and technological return can be performed by utilizing the Spacelab as a near-Earth plasma laboratory. Using the Spacelab as an in situ plasma laboratory and the ionosphere as a "working" rarefied plasma combines the experience and methods employed in space, with the practices and methods of controlled experimentation used in plasma chambers. This in situ experimental effort should be supported by an appropriate laboratory effort in conjunction with relevant theoretical studies, which investigate the flow field patterns around the bodies, i.e. the density discontinuities, the potential gradients, the temperature gradients and magnetic field fluctuations and time dependent phenomena. At the present time such theories are not available. 


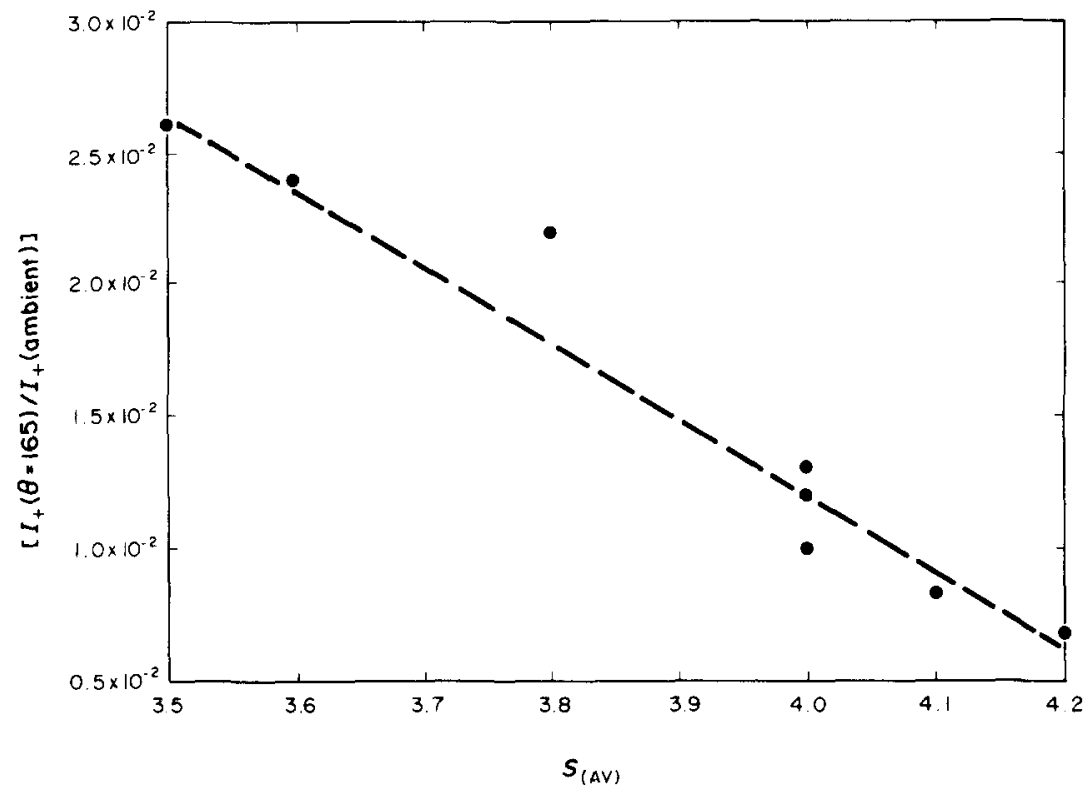

Fig. $9\left(\right.$ b). Variation of $\left[I_{+}(\theta=165) / I_{+}(\right.$ambient) $]$with average ionic Mach number $S_{(A V)}$ in the wake of the Explorer 31 Satellite (Data after Samir et al., 1973).

\section{Scientific and technological goals and methods}

The experimental goal of such a study is to measure in a parametric manner the local electron and ion density, local field potential, magnetic fluctuations, temperature enhancements and depletion and frequency in the ranges for excited waves; all in the near and far fields around a variety of objects. This should yield information relevant to the understanding of the specific conditions required for the creation of shocks ahead of and behind the objects, trailing expansion and compression waves, density enhancements and depletions, accelerating mechanisms, wave-particle interactions in localized potential wells, etc. and can help test the various flow interaction theoretical models. The objects (or bodies) to be used in the experimental study should differ in their geometry, surface properties, surface potential and the values and orientations of their intrinsic magnetic fields to the flow direction. Also, bodies should be used whose outer surface is made to evaporate, thereby creating bodies which are surrounded by an "atmosphere". The examination of the flow field patterns around such bodies for plasma with different properties and parameters should contribute to our understanding of the physical validity of applying gas-dynamic, kinetic and fluid approaches in describing collisionless plasma flows over natural and artificial bodies in space. Measuring the flow patterns around non-magnetized bodies with and without evaporating surfaces, for example, may in some respects partially 


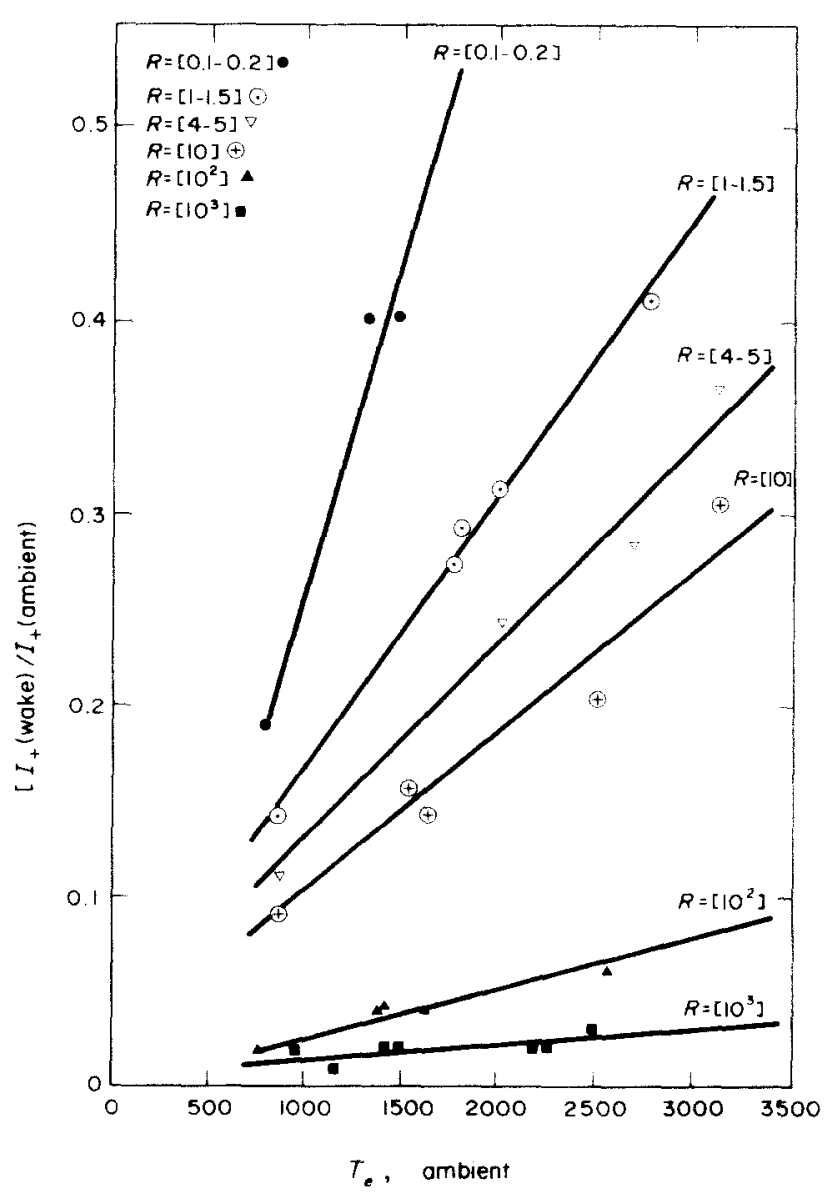

Fig. 9(c). Variation of $\left\{\left[I_{+}\left(\theta=160^{\circ}\right) / I_{+}\right.\right.$(ambient $\left.\left.)\right]\right\}=f\left(R_{D}\right)$ Atmosphere Explorer $C$ data (After: Samir et al., 1979).

"simulate" the flow around comets, Venus, the Moon or any other nonmagnetized bodies in the solar system as well as "simulate" the flow around artificial satellites moving in planetary environments. Despite the achievements of laboratory studies discussed earlier, these studies are still subject to technical limitations such as small scale size wall effects, etc. This argument is true for laboratory work attempting to simulate the interaction of objects in the solar system as well as to the area of satellite-ionosphere interactions. Moreover, the opportunity to perform experiments on board Spacelab in the near-Earth environment allows access to plasma regions whose properties approach the assumption typically made in plasma theory, e.g. of an infinite, homogeneous, fully ionized collisionless magnetoplasma. The present state of knowledge, both theoretical and experimental (in situ) was discussed in detail earlier. 
In the following section we will discuss methods of implementing experiments for the Shuttle/Spacelab where the test body ranges in size from 1 to $100 \mathrm{~m}$ in radius and range in complexity from a passive conductor to bodies with controllable electric and magnetic characteristics. The intent of this series of experiments is to use the unique capabilities of the Shuttle to investigate the full range of plasma flow interactions (PFI's) available for study within the ionosphere. Hence, various cases should be studied where the body radius is comparable to different plasma parameters, e.g. the Debye length, $\lambda_{D}$, and the ion Larmor radius, $L_{i}$. At least four ranges of size are of particular importance, i.e. $R_{0} \approx \lambda_{D}$, $\lambda_{D} \ll R_{0} \ll L_{i}, R_{0} \approx L_{i}$ and $L_{i} \ll R_{0}$. One would anticipate that the PFIs will take on different characteristics and result from different physical processes in the different ranges. Further, the question remains open as to when and under what conditions are theoretical treatments and approaches of a continuum nature no longer valid and hence a kinetic approach is to be used. According to Dryer (1970), the ratio $\left(R_{0} / L+\right)$ is analogous to the Knudsen number in fluid mechanics and can be used to determine whether a continuum or a kinetic approach must be used in theoretical treatments $\left(R_{0} / L+\gg 1 \rightarrow\right.$ continuum approach, $R_{0} / L+\ll$ $1 \rightarrow$ kinetic approach). It has been noted (Wu and Dryer, 1973) that a "transition" range exists for bodies such as the Earth's Moon, where $\left(R_{0} / L+\right)$ does not provide a decisive answer. Therefore, experiments in the "transition" region (which can be studied in the ionosphere) are important; particularly since they cannot be performed effectively in the laboratory.

In addition to their importance to the investigation of basic rarefied plasma physics, the experiments conducted in the range $R_{0} \approx \lambda_{D}$ and $\lambda_{D} \ll R_{0} \ll L^{+}$lend themselves to the practical problems of instrument and spacecraft interactions with the space plasma. The large body experiments, where $L+\gg R_{0}$, may well have a bearing on the interaction of the solar wind with certain types of planetary bodies. The inclusion of an orientable magnetic dipole of variable strength may further extend the application to solar wind interactions with bodies having an intrinsic magnetic field and may improve our understanding of physical processes such as magnetic field line merging. As discussed in an earlier section, such applications will rely heavily on the concept of qualitative scaling.

In summary, the scientific and technological objectives can be grouped as follows:

Group 1. Study of flows over bodies in the terrestrial atmosphere-ionosphere-magnetosphere for the purpose of understanding the interaction between a spacecraft and a planetary environment and for testing theoretical models. This group consists of both scientific and technological goals.

Group 2. Study of model experiments relevant to flow interactions of space plasmas with planets and their moons. Such studies will be of particular interest to the supersonic and sub-Alfvenic flow regimes (e.g. motion of Io around Jupiter). Such studies could also be of relevance to the development of a unified theory of planetary magnetospheres. Here the principle of qualitative scaling should be utilized and the model experiments would be of the process simulation type. Experiments in this group will probably have application to plasma astrophysics and cosmic gas dynamics. They may also be of value to the 
examination of concepts which are in various stages of development. Such studies could help evaluate the degree of applicability of such concepts to phenomena in space (Falthammar, 1974).

The Spacelab/Orbiter as a rarefied plasma laboratory for performing controlled plasma flow experiments in space-general discussion on modes of operation

Among the unique capabilities of Spacelab most applicable to experimental studies of plasma flow interactions with objects in space are: (1) The availability of multiple flights which provide for flexible complements of reusable core instruments that could allow for the simultaneous measurements of a large number of plasma parameters. (2) The availability of booms which can provide for the desirable mounting of various interchangeable test-bodies and instrument ensembles. The booms can be made to rotate articulately in space. The controlled maneuvers so employed allow for a large degree of flexibility in experimental planning and in selecting optimal modes of operation. Boom maneuvers can be pre-planned with capability of in-flight modifications based on the "quick examination" of preliminary data received on board. The "maneuverable boom" capability allows for comprehensive and extensive measurements of yet unexplored zones around the "test bodies". (3) The availability of subsatellites and small instrument packages which can be ejected from the Spacelab immensely enhances the spatial extent around the "test bodies" that can be investigated; particularly if the subsatellite is maneuverable. (4) The large payload capability of the Spacelab makes it possible to interchange many "test bodies" of different sizes, geometries and body properties, and study the flow patterns around them in a parametric manner. Various properties of the "test bodies" could be varied in flight thus allowing for a wide scope of spatial and temporal comparisons. By using bodies of well defined geometries and surface properties, various theoretical models could be tested hence assessing in an unambiguous manner the validity of assumptions used in these theories. (5) The capability of real time examination of data from displays on board the Spacelab, hence allowing the crew to perform changes in the experimental operations. A class of "test bodies" of particular interest is that known as the "tetheredsatellite-system" (TSS). Large tethered bodies accompany the Spacelab/Orbiter in flight, and can thus act as wake/sheath creating bodies. It appears that scientists from various disciplines of space science and technology are interested in performing experiments utilizing such a system. This group of "test bodies" seems particularly suitable for studies where $\left|\left(R_{0} / \lambda_{D}\right)\right|$ and $\left|\left[R_{0} / R_{L}(+)\right]\right|$ have to be larger than $10^{3}$ and $10^{1}$, respectively. This allows systematic experimental studies in the supersonic and sub-Alfvenic flow regime with applications to phenomena and situations in the solar system and deeper space. As mentioned earlier, the probes used in "wake and sheath" studies in the past were mostly surface mounted and hence a priori limited to measurements of the very near vicinity of the satellites. Thus, trailing shocks behind and shocks ahead of the satellites, distant density gradients around the satellite, the location of potential wells and other prominent structures could not have been observed.

According to the suggested approach the Spacelab/Orbiter (Fig. 10) is in- 


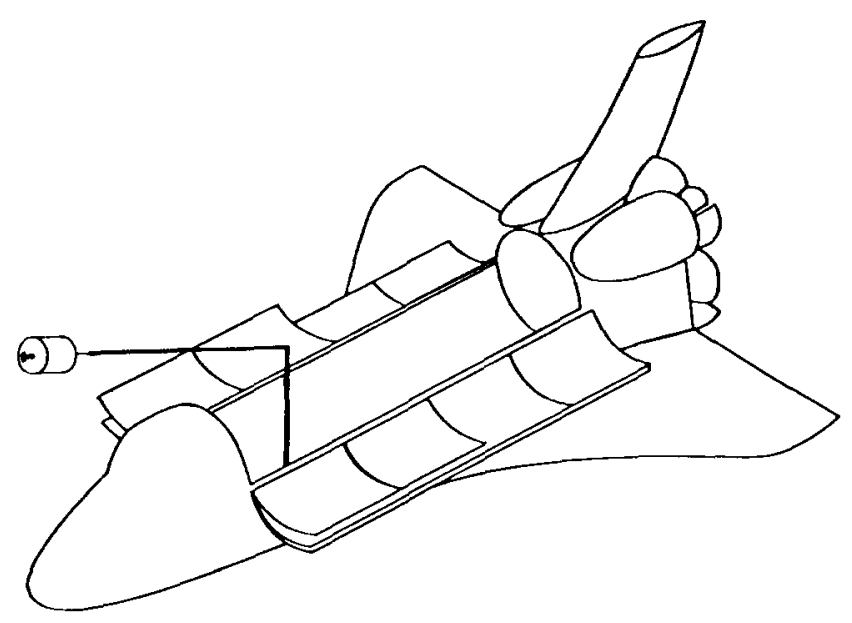

Fig. 10. A schematic drawing of the Spacelab/Orbiter (including a boom).

jected into orbit and carries the appropriate ensembles of instruments and the "test bodies" on board. The "test bodies" should range in complexity, depending on the experimental objectives for specific missions, beginning with spherical and cylindrical conductors having similar cross-sections exposed to the flow and proceeding to more complex bodies with controllable electric characteristics (such as body potentials). This could then furnish a wide range of variation for the parameter $\left|e \phi_{N}\right| K T_{e} \mid$. This parameter, as is known, is very important in its influence on the flow structure around the test body (Al'pert, 1976; Parker, 1977). The test bodies should vary in their linear dimensions, hence providing for a large range of the parameter $R_{D}\left[=\left(R_{0} / \lambda_{D}\right)\right]$. It would be preferable in early experiments, to use bodies of the same geometry but having different crosssectional areas.

More advanced test bodies should follow and should include experimental arrangements for creating a dipole magnetic field with the capability of controlling its strength and orientation with respect to the velocity vector. Such investigations will have potential applications to geo/astrophysics. In addition the spectra of test bodies should include those whose outer surfaces evaporate upon plasma impact, thereby producing a gas cloud surrounding the body.

A simple experimental arrangement for measuring the charged particle density and temperature, local potential and magnetic field around a well defined "test body" is shown in Fig. 11. To perform experiments in this mode two booms (Fig. 11a) or one boom and a fixed stem (Fig. 11b) are required. The booms can be maneuverable and/or fixed and their length will depend upon the specific scientific and/or technological objectives of the investigation. Another consideration which will enter into the definition of boom length and other boom characteristics will be the spatial extent of the interference due to the Spacelab/Orbiter itself. It is quite clear therefore, that the disturbances created by the Spacelab itself will have to be investigated (at least partially) prior to beginning the experimental program discussed here. 


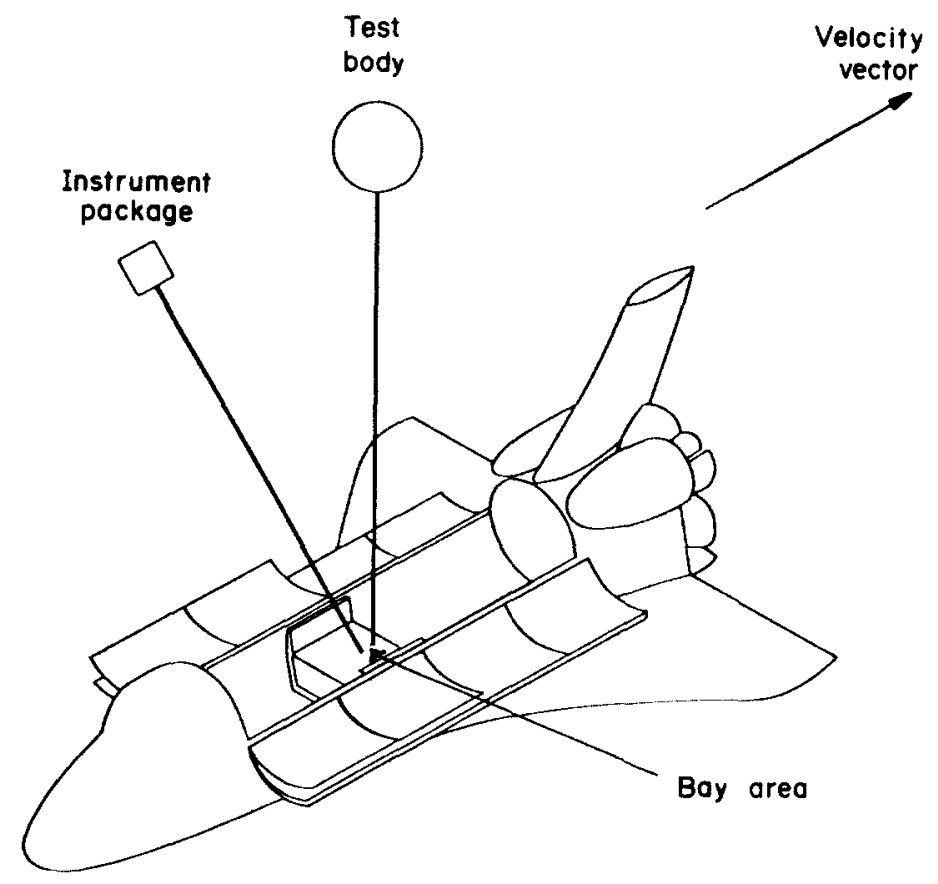

Fig. 11(a). The Spacelab/Orbiter with two maneuverable booms. One holding the "target-body" and the other holding the instrument package.

When subsatellites and/or throw-away-detectors (TADs) become available for specific Spacelab missions it will be possible to eject the test body and have the instrument package mounted on a boom. In this mode of operation the test body is a "free-flyer". The general flight configuration is shown schematically in Fig. 12. A wide and flexible range of pre-planned trajectories in the vicinity of the Spacelab/Orbiter can be implemented for the ejected TADs and subsatellites by providing the appropriate initial velocity and injection angle. A practical example of such a pre-planned trajectory is given in Fig. 13, which shows the computed spatial position of the TAD or subsatellite with respect to the Spacelab/Orbiter. Some details on this example are given in Table 10. The Spacelab itself can be made to torate around selected axes of rotation as shown in Fig. 14. Rotation and maneuverability of the Spacelab/Orbiter itself provides for more flexibility in selecting the mode of operation and flight configuration.

In addition to test bodies mounted on booms and free flyers, test bodies can also be utilized in the form of tethered balloons (instrumented and non-instrumented). The use of large inflatable balloons in the tethered mode of operation, will simplify technical difficulties involved in obtaining large values for the parameters: $\left|\boldsymbol{R}_{0} / \boldsymbol{R}_{L(+)}\right|$ and $\left|R_{0}\right| \lambda_{D} \mid$. Figure 15 provides some possible subsatellite mappings for target bodies with effective radii of $R_{0}=1 \mathrm{~m}, 10 \mathrm{~m}$ and $100 \mathrm{~m}$. Relevant details are given in Table 11.

As discussed earlier, the plasma flow interactions with bodies that are large 


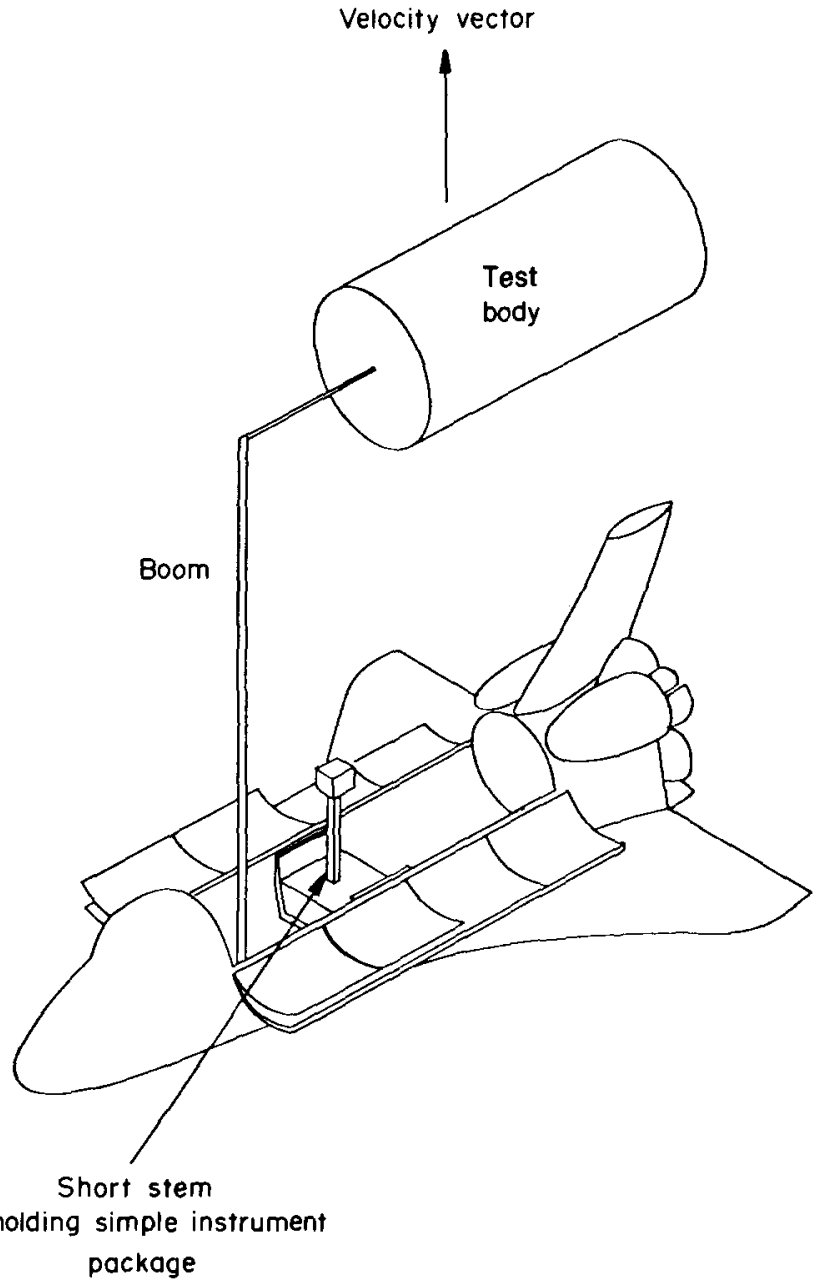

Fig. 11(b). The Spacelab/Orbiter with a large cylindrical body (on a fixed boom) and a short stem holding a simple instrument package.

Table 10. Parameters associated with the maneuver

\begin{tabular}{|c|c|c|c|c|c|}
\hline \multirow[b]{2}{*}{ Arc } & \multirow[b]{2}{*}{ Time(sec) } & \multirow[b]{2}{*}{$\Delta V(\mathrm{~m} / \mathrm{sec})$} & \multirow[b]{2}{*}{ Propellant(lb) } & \multicolumn{2}{|c|}{$\begin{array}{l}\text { Maximum possible error in } \\
\text { pos at end of arc }\end{array}$} \\
\hline & & & & $\Delta X$ & $\Delta Y$ \\
\hline 1 & 250 & 0.809 & 57 & $\pm 1 \mathrm{~m}$ & $\pm 3.5 \mathrm{~m}$ \\
\hline 2 & 100 & 2.887 & 203 & $\pm 4 \mathrm{~m}$ & $\pm 7 \mathrm{~m}$ \\
\hline 3 & 375 & 2.978 & 209 & $\pm 10 \mathrm{~m}$ & $\pm 11 \mathrm{~m}$ \\
\hline 4 & 141 & 1.918 & 135 & $\pm 15 \mathrm{~m}$ & $\pm 15 \mathrm{~m}$ \\
\hline
\end{tabular}


(a)
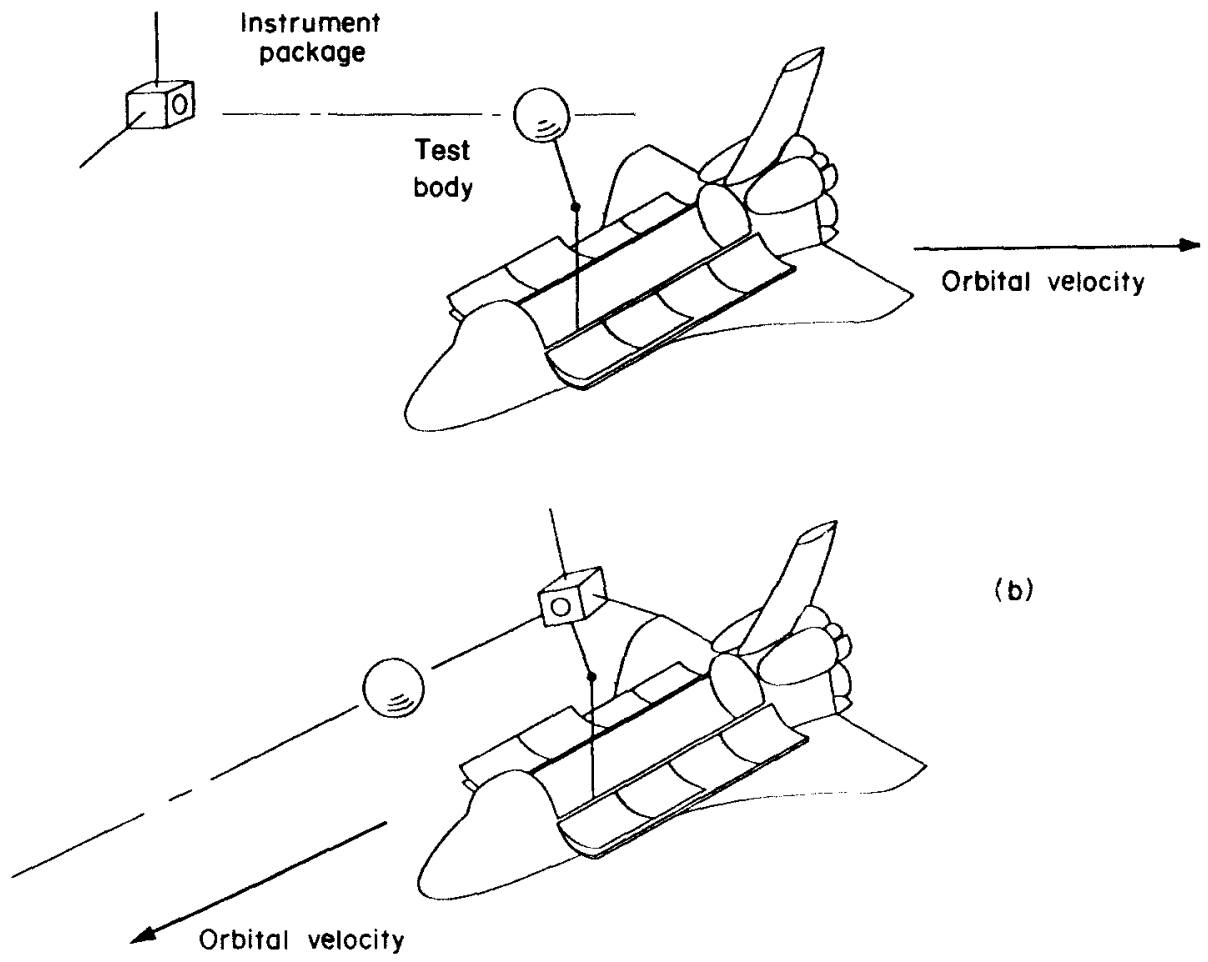

(b)

Fig. 12. (a) The Spacelab/Orbiter with target body mounted on boom and ejected instrument package acting as a "free flier". (b) The Spacelab/Orbiter with role of target body and instrument package interchanged.

Table 11. Sub satellite mapping parameters

\begin{tabular}{lccc}
\hline Parameter & $R_{0}=1 \mathrm{~m}$ & $\boldsymbol{R}_{\mathbf{0}}=10 \mathrm{~m}$ & $\boldsymbol{R}_{\mathbf{0}}=100 \mathrm{~m}$ \\
\hline Time & 600 & 600 & 600 \\
Total distance (m) & 190 & 885 & 5035 \\
Av. velocity (m/sec) & 0.32 & 1.48 & 8.39 \\
Max. velocity (m/sec) & 0.32 & 1.55 & 8.73 \\
Meas. duration (sec) & 0.5 & 0.5 & 0.5 \\
Spatial resolution (m) & 0.16 & 0.78 & 4.37 \\
No. firings & 27 & 20 & 21 \\
Total $\Delta v$ (m/sec) & 8.64 & 31.0 & 183.3 \\
Acceleration distance (m) & 2.0 & 15.0 & 200.0 \\
Acceleration time (sec) & 6.32 & 10.17 & 23.83 \\
Assumed Sub.Sat.Mass ${ }^{\prime}(\mathrm{kg})$ & 0.05 & 0.15 & 0.37 \\
Acceleration (m/sec ${ }^{2}$ ) & 657.6 & 657.6 & 657.6 \\
Thrust Newtons & 33.3 & 100.2 & 240.7 \\
\multicolumn{1}{c}{$(\mathrm{lb})$} & $(7.5)$ & $(22.5)$ & $(54.1)$ \\
\hline
\end{tabular}

$\dagger$ Assumed mass is that at an AE satellite $-1450 \mathrm{lb}$. 


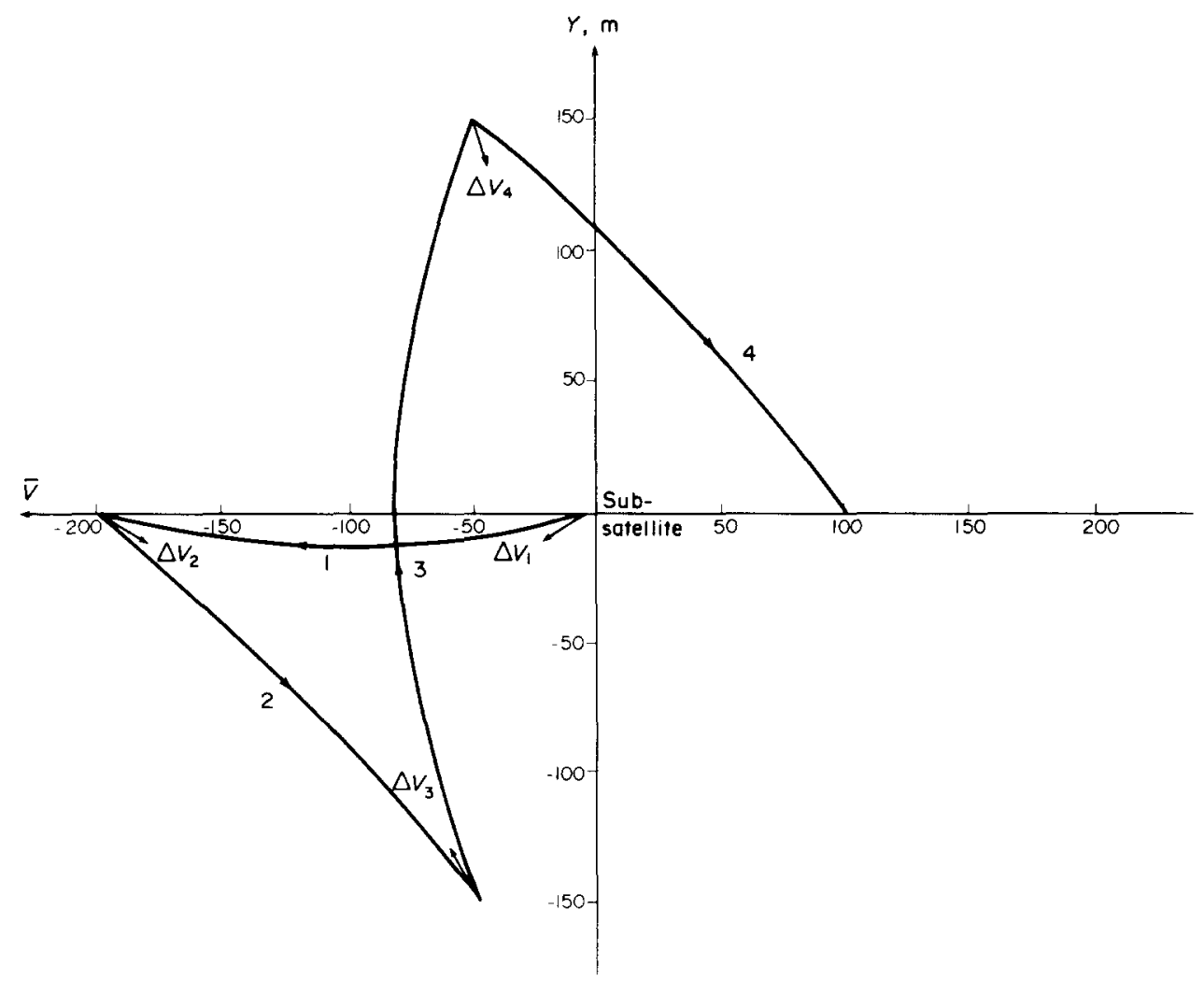

Fig. 13. An example of a Spacelab/Orbiter and a subsatellite maneuver. The figure shows the relative position in space between the subsatellite and the Orbiter.

compared to $\lambda_{D}$ or $L_{+}$may be expected to be physically different, than for small bodies and consequently require a different theoretical approach (Parker, 1976, 1977; Kunemann, 1978). Further, such interactions may shed light on certain celestial interactions. As examples, we will consider the interaction of the Moon with the solar wind and that of Io in Jupiter's environment.

From the scaling parameters given in Table 12, we find that the body should consist of a spherical balloon and range in size up to $300 \mathrm{~m}$ in diameter in order to provide the $\left(R_{0} / L_{+}\right)$value appropriate for the Moon in the solar wind. The surface characteristics should be varied for different experiments (conducting or insulating). For conducting bodies, provisions should be made to control the body potential. For large insulating bodies, provisions should be made to measure differential charging of the surface.

The variables that need to be measured as a function of space and time for the large body experiments are: the ion and electron temperatures and densities, the ion flow direction, flow velocity and mass, the space potential and electric fields. For the most part, standard diagnostic instruments, or instruments now under development, can provide these measurements. All measurements should be made by a mapping instrument package in the disturbed zone concurrent with 

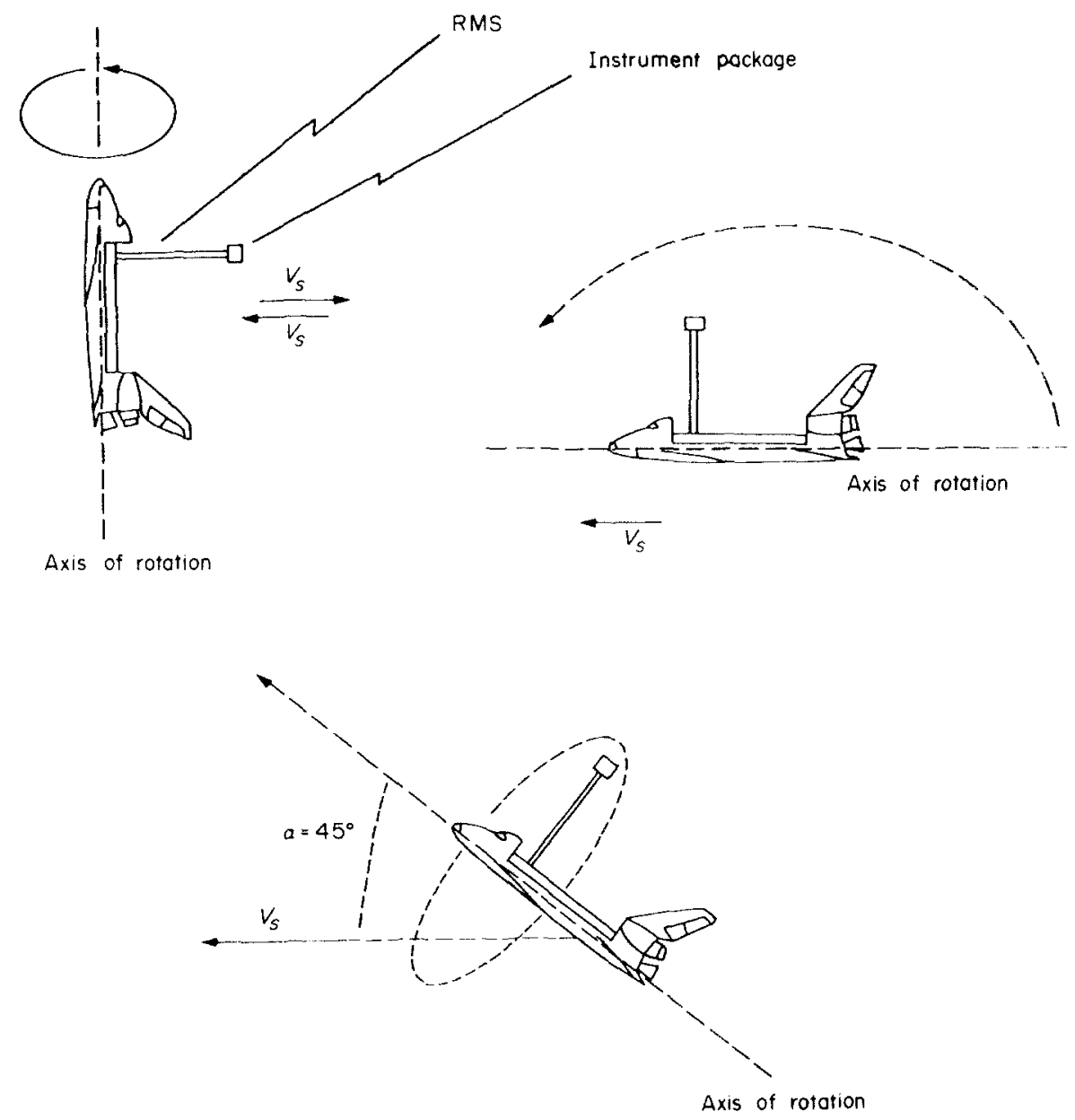

Fig. 14. The Spacelab/Orbiter performs rotations in orbit.

Table 12. Scaling parameters (after Stone and Samir, 1978)

\begin{tabular}{lcccccc}
\hline Body & $\begin{array}{c}\boldsymbol{R}_{\mathbf{0}} \\
(\boldsymbol{m})\end{array}$ & $S$ & $\frac{\boldsymbol{R}_{0}}{\lambda_{D}}$ & $\frac{\boldsymbol{R}}{L_{+}}$ & $\frac{\boldsymbol{R}_{0}}{L}$ & $\boldsymbol{R}_{M}$ \\
\hline Moon & $1.74 \times 10^{6}$ & 15 & $3 \times 10^{5}$ & 29 & $1 \times 10^{3}$ & $1 \times 10^{10}$
\end{tabular}

\section{Balloon}

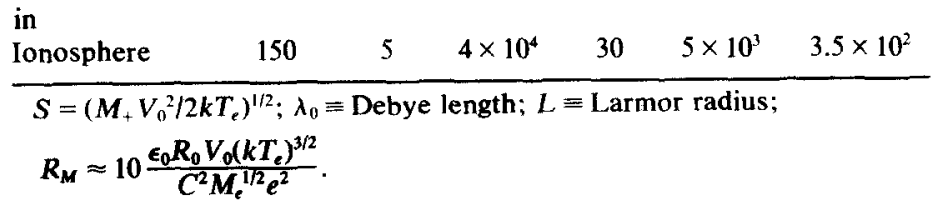




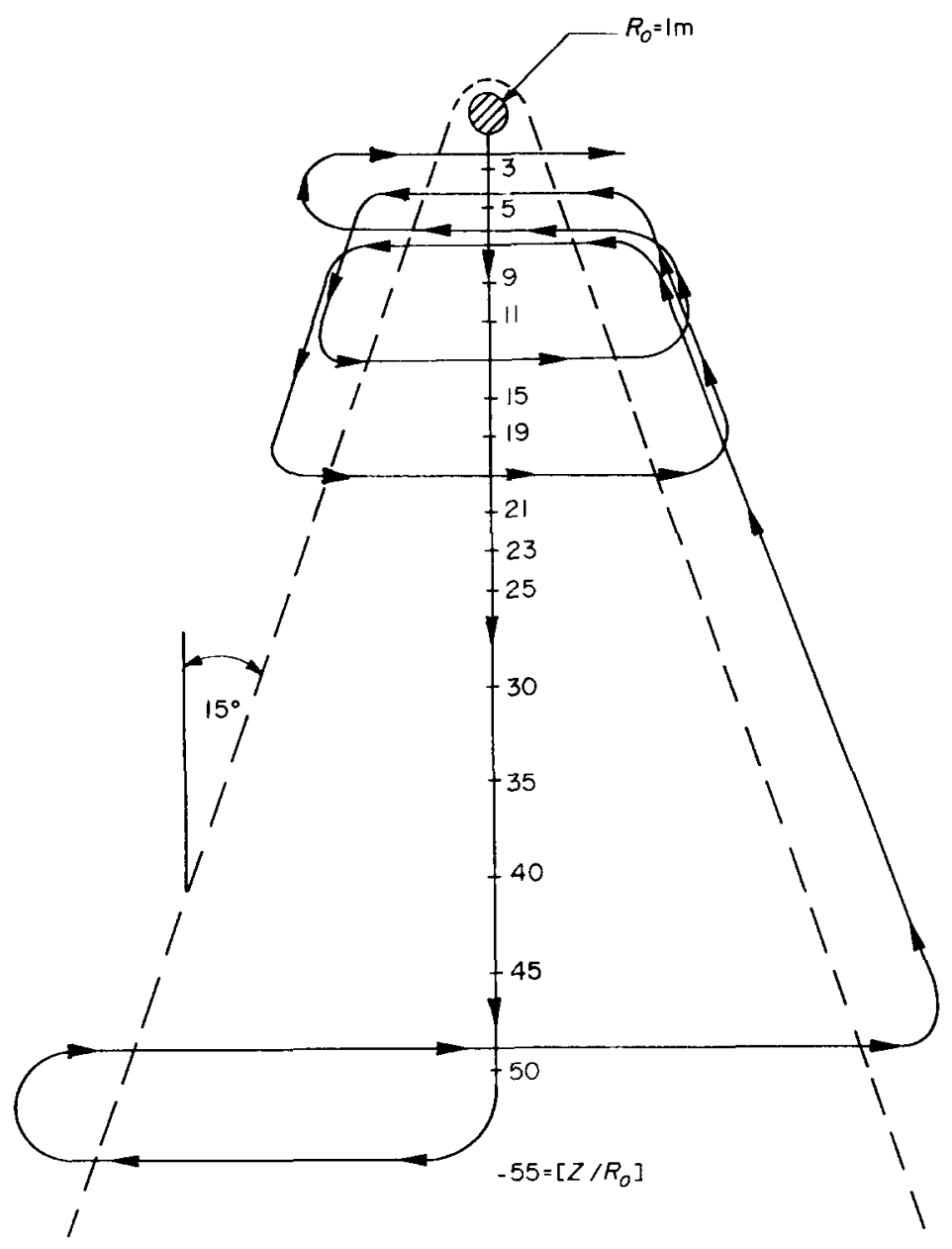

Fig. 15(a). Examples for pre-planned subsatellite mappings, around "test bodies" with

$$
R_{0}=1 \mathrm{~m} \text {. }
$$

a complete set made in the ambient medium. This is necessary to separate temporal and spatial fluxations generated by the body from those naturally occuring in the ambient medium.

The spatial volume of interest consist of a small frontal region where a bow shock may exist under certain circumstances, and the downstream wake. The extent of these regions is not accurately known for large bodies and the definition of the limits of the disturbed zone should be the object of early experiments. Since, for such large bodies, the sheath fields are not expected to have an important influence, the ion void swept out by the moving body may be expected to fill by thermal or other processes such as ambipolar diffusion. The disturbance can therefore be expected to be confined to a "Mach" angle defined in terms of the ion acoustic speed. This is typically less than $20^{\circ}$ in the lower ionosphere. Figure 16 shows a conceptual arrangement of the balloon and the 


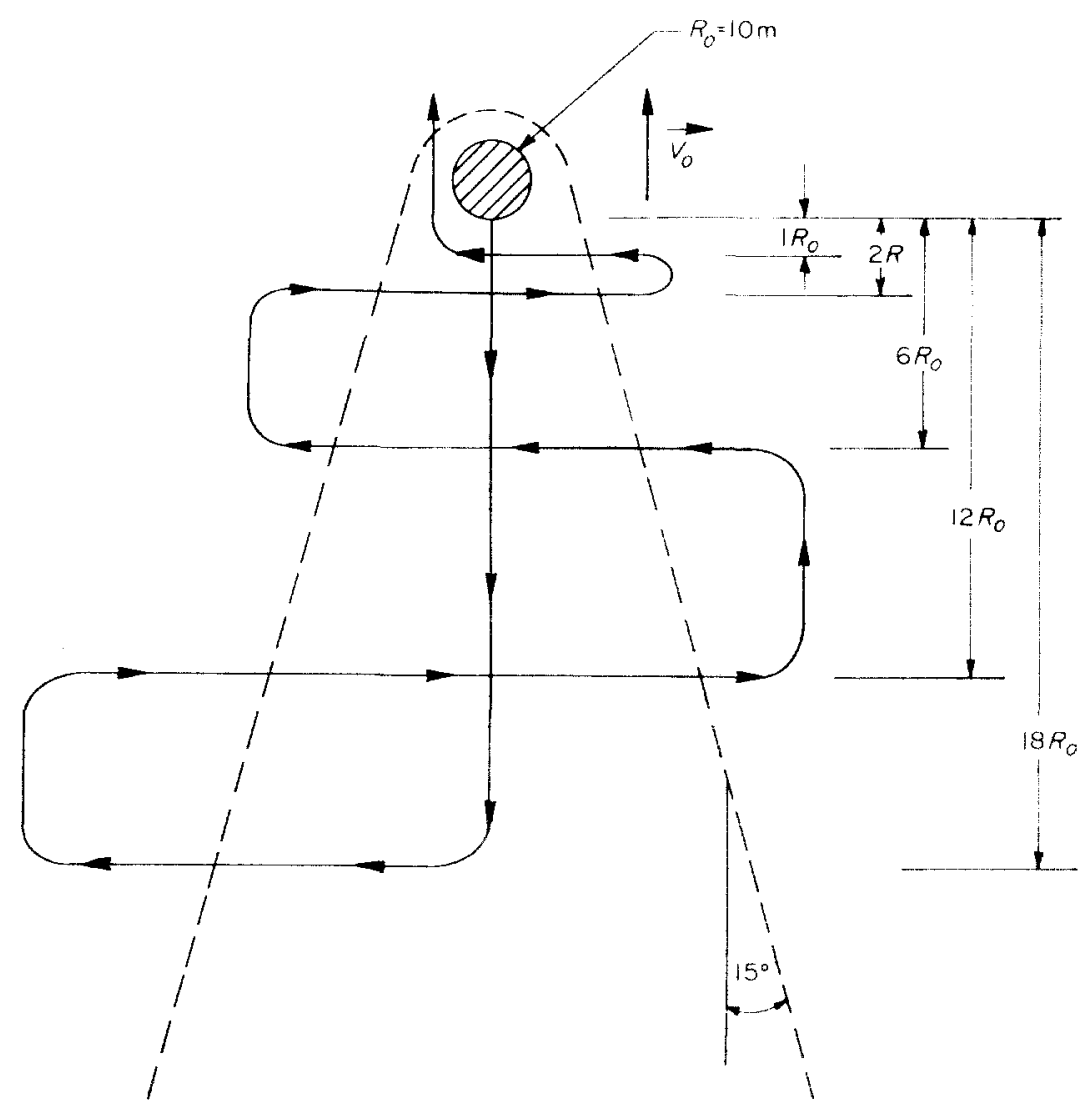

Fig. 15(b). Examples for pre-planned subsatellite mappings around "test bodies" with $R_{0}=10 \mathrm{~m}$.

mapping and ambient monitoring instrument packages using the Tethered Satellite System (TSS). Figure 15(c) shows an example for the mapping maneuver for investigating the frontal and near wake regions. In order to maintain consistent conditions, it is desirable that such a maneuver be completed within one orbit in a region where ionospheric conditions are relatively constant (i.e. avoiding the polar regions and the terminators). This would require that the mapping be completed in approx. $15 \mathrm{~min}$.

The primary purpose of the tethered satellite system in large body studies is to deploy the test body and maintain its position with respect to the Orbiter. As indicated in Fig. 16 it may also be used to deploy an ambient monitor.

The fact that a tether is, indeed, required to maintain bodies as large as indicated in Table 12 is graphically demonstrated in Fig. 17. This shows the separation distance, produced by differential drag between the Spacelab/Orbiter and large balloon type bodies, as a function of time and body radius at $400 \mathrm{~km}$ altitude. The maximum time shown is 15 min which is the desired length of the mapping maneuvers. After this time, the $20 \mathrm{~m}$ body is still within a few 


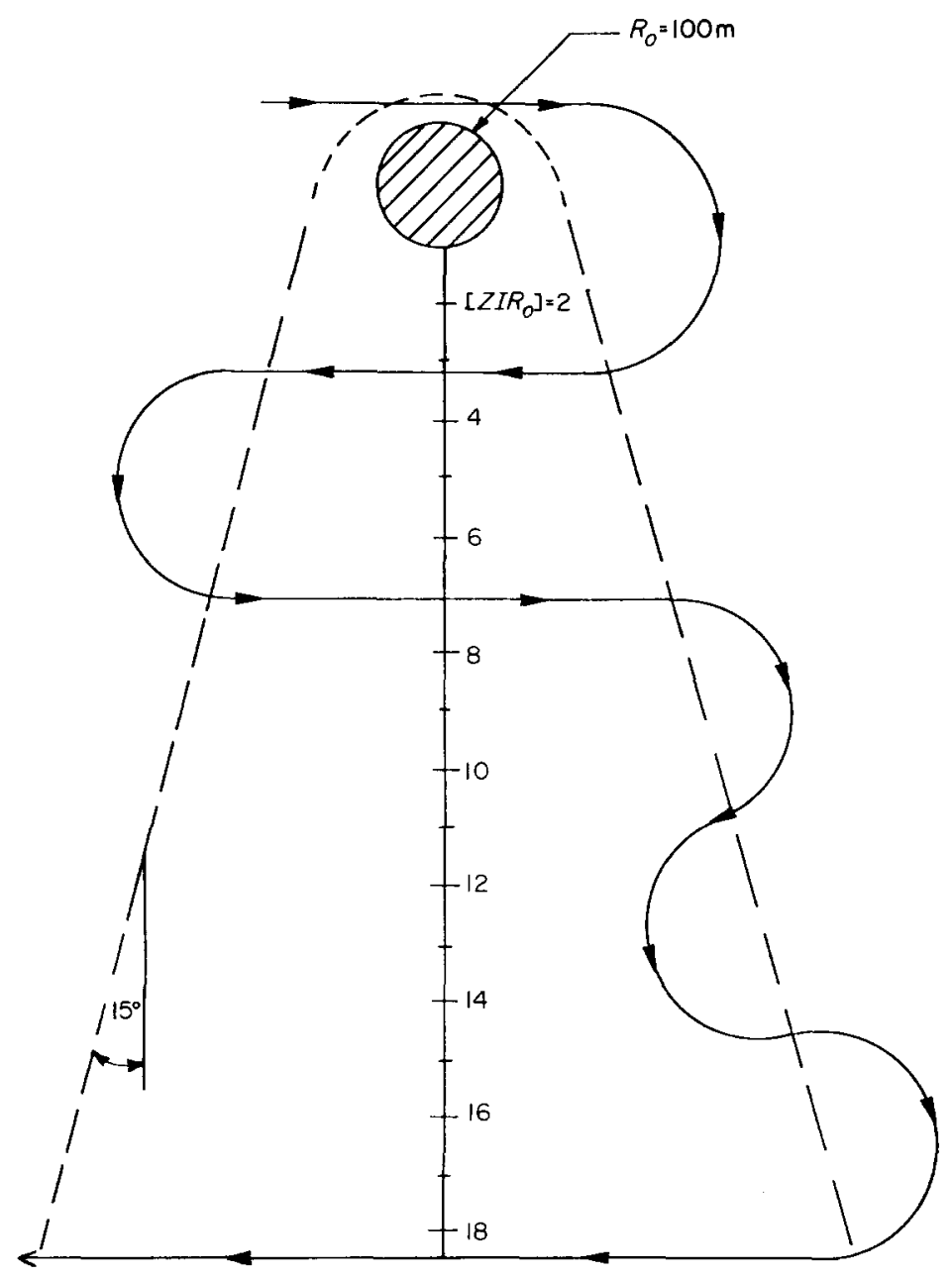

Fig. 15(c). Examples for pre-planned subsatellite mappings, around "test bodies" with $R_{0}=100 \mathrm{~m}$ (after Stone and Samir, 1978).

kilometers of the Orbiter, which is permissible if only one mapping is required. However, re-use would require repositioning, possible by a maneuverable subsatellite. For the larger bodies of $100-200 \mathrm{~m}$ in radius, the separations grow to the order of $10^{2} \mathrm{~km}$, which is not practical, even for a single mapping maneuver. The acceleration of two bodies can be matched by equating their ballistic coefficients (Mulling, 1975), however, for balloons with $R_{0}>50 \mathrm{~m}$, this would require a ballast mass in excess of $10^{6} \mathrm{~kg}$ and is therefore not practical. Therefore, for bodies of this size the tether becomes essential for station keeping.

Having shown the tether to be necessary for the large body experiments, it remains to be determined if tethered balloons of this size are practical-Is the drag on the balloon excessively great?-Is the system stable?-What magnitude 


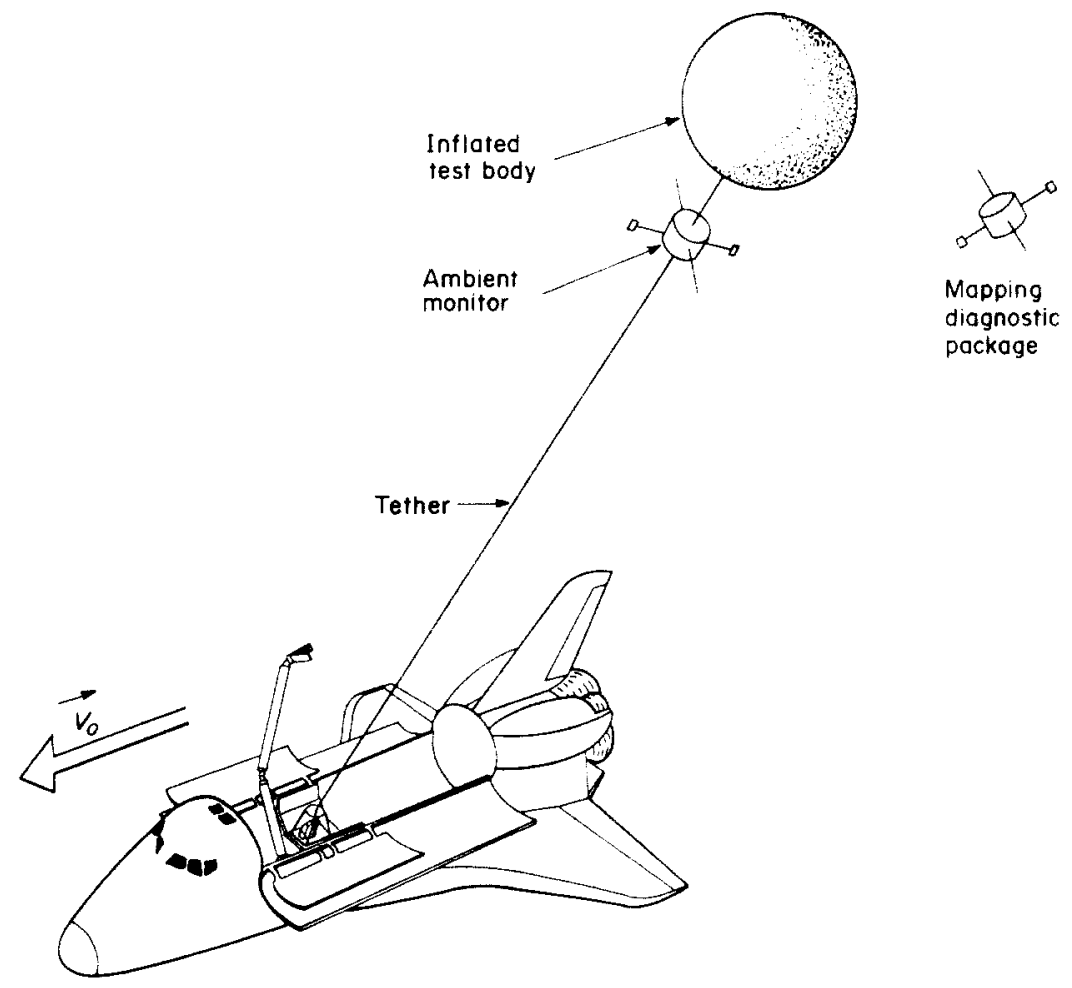

Fig. 16. Schematic drawing showing the use of a tethered inflatable balloon (after Stone and Samir, 1978).

of current must be handled by the tether to implement the voltage control discussed above?

Figure 18 shows the drag force $F_{D}$ on balloons of several different radii, $R_{0}$, as a function of altitude. From the report by Rupp and Laue (1978), we take a tether tension of $100 \mathrm{~N}$ to be the current design criteria. As expected, the figure shows that the drag becomes large rapidly with decreasing altitude, and, certainly below $200 \mathrm{~km}$ large body experiments are not practical. It is therefore immediately obvious that only an upward deployment relative to the Shuttle can be used.

The exact range of permissible balloon radii and altitudes depends on the maximum allowable angle the TSS can make to the vertical. This was not given in the reports on the TSS although angles of $50^{\circ}$ are shown (Rupp and Laue, 1978). We assume $45^{\circ}$ to be a reasonable design criteria and take the satellite mass to be $175 \mathrm{~kg}$ (the design value given by Rupp and Laue). Then a balloon with $R_{0}=150 \mathrm{~m}$ can be deployed as low as $400 \mathrm{~km}$. Therefore, from these initial drag considerations, the size of bodies in the $R_{0} \sim 10^{2} \mathrm{~m}$ range is not a problem.

The primary concern in biasing the balloon is handling the current collected, either by conduction to the Orbiter through the tether or by charged particle emission at the balloon. Most PFI experiments will probably involve negative 


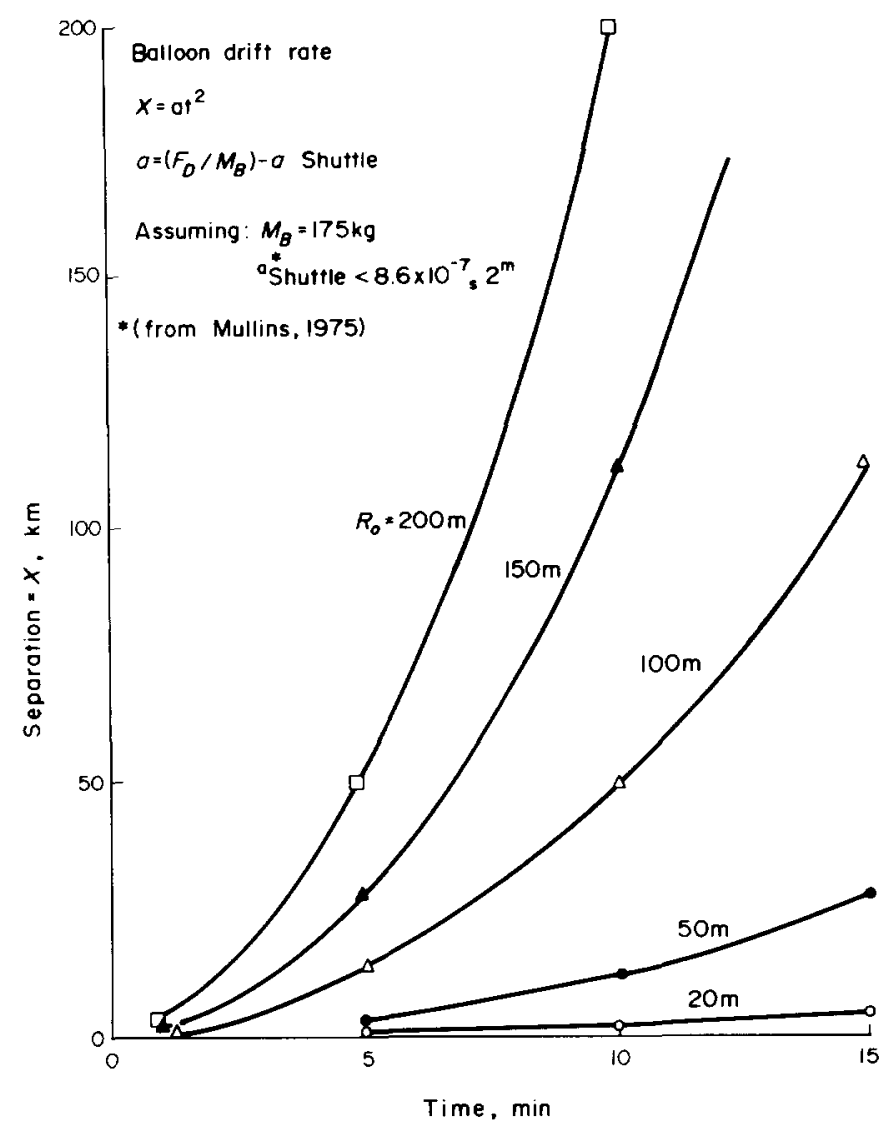

Fig. 17. Separation distance vs time and balloon radius for a Spacelab/Orbiter and a balloon experiment (see Fig. 16 for flight configuration) (after Stone and Samir, 1978).

biasing, and hence, the collection of a net positive ion current. According to Williamson and Banks (1976), the body potential is not a major factor in the collection of electrons (only $\sim 14 \%$ enhancement for a $2 \mathrm{kV}$ bias). It is therefore reasonable to expect the positive ion current to be essentially independent of body bias. The positive current collected by the balloon will therefore consist primarily of the ion flux swept out by the balloon's movement. The resulting current is shown in Fig. 19 as a function of altitude for several balloon sizes. There does not appear to be any difficulty with negative biasing with the possible exception of the 150 and $200 \mathrm{~m}$ radii balloons at $400 \mathrm{~km}$ altitude. However, the current drops off rapidly on either side of $400 \mathrm{~km}$ and this region could be avoided.

Positive biasing is expected to be more difficult to achieve. The actual current collected is more difficult to estimate, but if the thermal electron current can be taken as an indication, even at $300 \mathrm{~km}$ altitude, a balloon with $R_{0}=100 \mathrm{~m}$ would collect $\sim 50 \mathrm{amps}$. This value grows rapidly with increased balloon radius and altitude. 


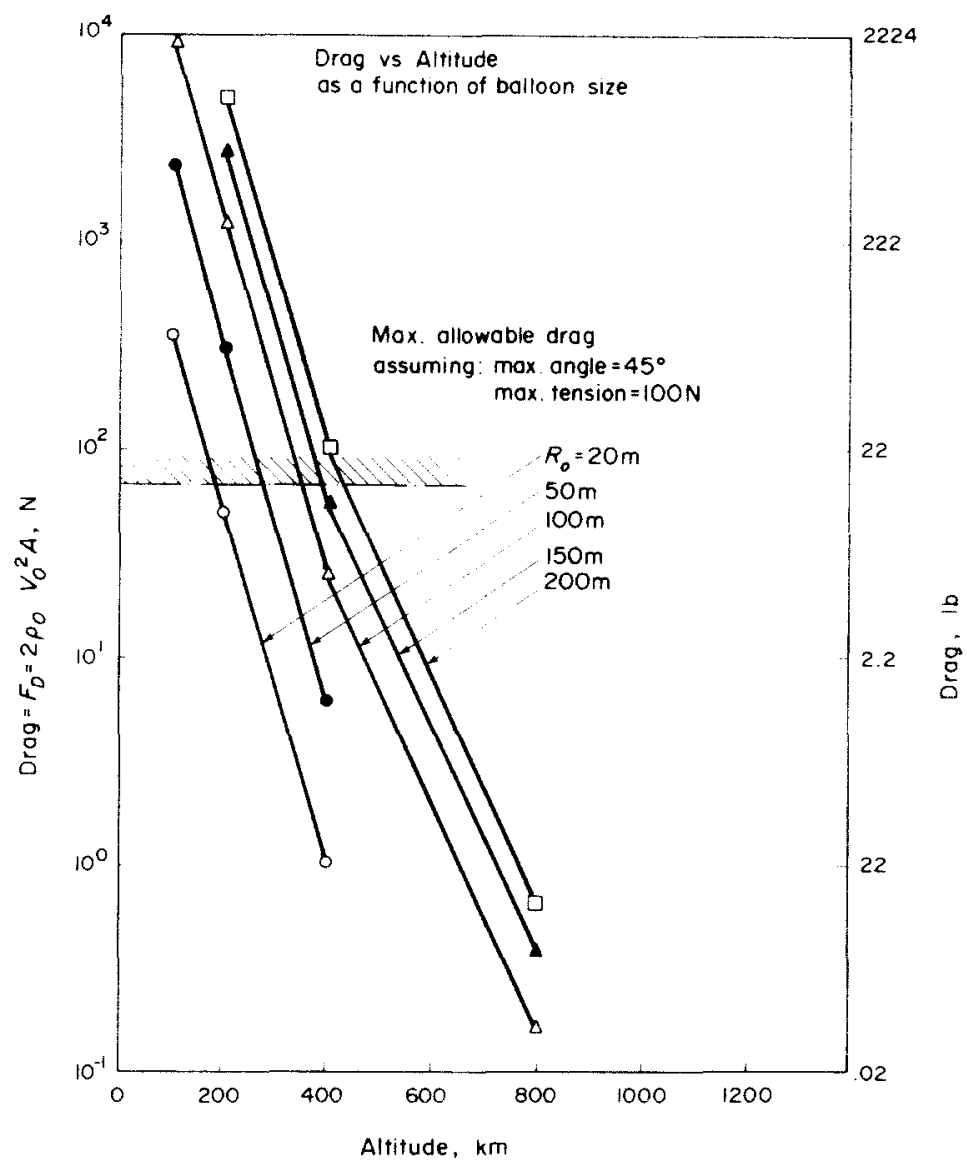

Fig. 18. The drag force on balloons of several different radii as a function of altitude (after Stone and Samir, 1978).

The deployment rate of the TSS is in the range of $10-50 \mathrm{~m} / \mathrm{sec}$. This is sufficient to provide a large part of the required maneuvering capability represented by Fig. 15(c). Since the trajectory lengths across the disturbed zone are on the order of $0.5-1.5 \mathrm{~km}$, a single traverse could be carried out typically in one or two minutes. The critical question is what kind of undesirable gyrations of the balloon, if any, would be initiated by such maneuvers.

About the feasibility of using the Spacelab as a "rarefied plasma laboratory" for plasma flow interactions studies-technological comments

Prior to the utilization of the Spacelab/Orbiter as a rarefied plasma laboratory it is mandatory to obtain a realistic assessment of the magnitude and spatial extent of the disturbances due to the motion of the Spacelab/Orbiter itself. To achieve this technological goal the charged particle and potential distributions have to be measured at various locations in the vicinity of Spacelab and be compared with ambient (undisturbed) conditions. Also the spacecraft potential at several locations on the surface will have to be measured to get a realistic 


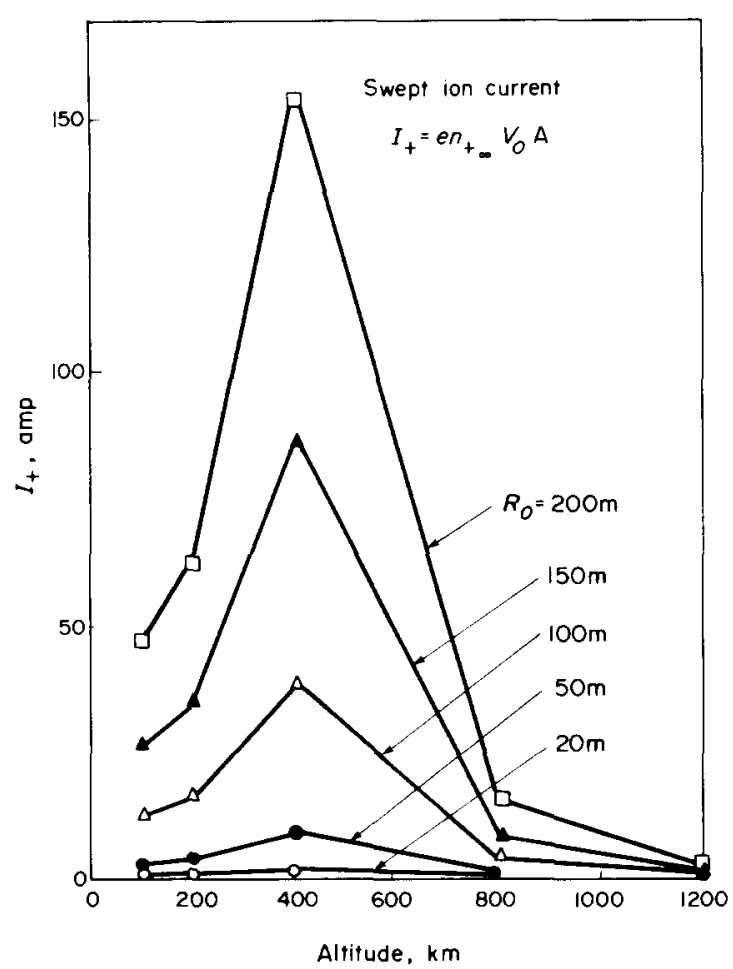

Fig. 19. Swept ion current as a function of altitude for balloons of different radii $\left(R_{0}\right)$ : $R_{0}=20,50,100,150$ and $200 \mathrm{~m}$ (after Stone and Samir, 1978).

assessment regarding effects connected with "spacecraft-charging". Such preliminary studies could yield preferential choices for types of instrumentation to be used and their location on Spacelab and may even rule out the use of common direct measurement devices. Such investigations should also help assess how long the booms should be for various flight configurations. The need to examine the perturbations created by the Spacelab/Orbiter is recognized by NASA as an essential technological forerunner to the scientific program for Spacelab.

The brief discussion of this section is devoted to the perturbation caused by the Spacelab motion to its environmental charged particle component. Electromagnetic interference (EMI), gas leakages and disturbances to the neutral component of the Orbiter's environment are not included in the discussion although the charged and neutral components are obviously coupled. We will show here how, in a relatively simple way, the technological objective mentioned above can be achieved. There are similarities between the modes of operation for the scientific program discussed earlier and the modes of operation required for achieving the technological goals.

For knowledge of the magnitude of the perturbations in the near vicinity of the Spacelab/Orbiter payload bay area it is sufficient to have a short stem (say, 
2-5 $\mathrm{m}$ in length) located in the bay area (see Fig. 10) which carries a simple instrument package adequate for measuring the local electron and ion density and temperature and the local plasma potential and magnetic field. In order to obtain an optimal amount of information the Shuttle/Orbiter should be made to undergo roll and pitch maneuvers at different angles of attack, as shown schematically in Fig. 14. In order to extend the spatial regions that could be mapped, a maneuverable boom which will hold the instrument package or the TAD should be used. NASA Announcements of Opportunities regarding Spacelab missions specify that such a boom known as an RMS (remote maneuvering system) will be available as part of the Spacelab system. Hence, the charged particle distributions and the local electric potential and magnetic field could be obtained for distances up to about $15 \mathrm{~m}$ from the surface (see Fig. 20). To achieve a good experimental coverage it is possible to perform two types of maneuvers: (a) with the RMS extended at a fixed distance and stationary, the orbiter undergoes roll and pitch maneuvers (see Fig. 14) and (b) with the orbiter in a fixed orientation, the RMS probes within the region accessible to it (see Fig. 21). In order to significantly extend the spatial regions to be probed, the instrument package or TAD should be spun and ejected and hence, operate as a free flier around the Spacelab/Orbiter. Once the TAD is ejected, the Orbiter could be made to maneuver along a specified trajectory to obtain a useful mapping of the disturbed zone at distances, ranging from $50 \mathrm{~m}$ to several hundreds of meters from its surface. A simple example for such a pre-planned orbiter/subsatellite (or TAD) maneuver at an altitude of $200 \mathrm{n} . \mathrm{m}$. and for density and drag of $p-1.05 \times 10^{-11}\left(\mathrm{~kg} / \mathrm{m}^{3}\right)$, and $D=9 \times 10^{-9}\left(\mathrm{~m} / \mathrm{sec}^{2}\right)$ respectively is shown in Fig. 13. Some parameters associated with this maneuver are given in Table 10.

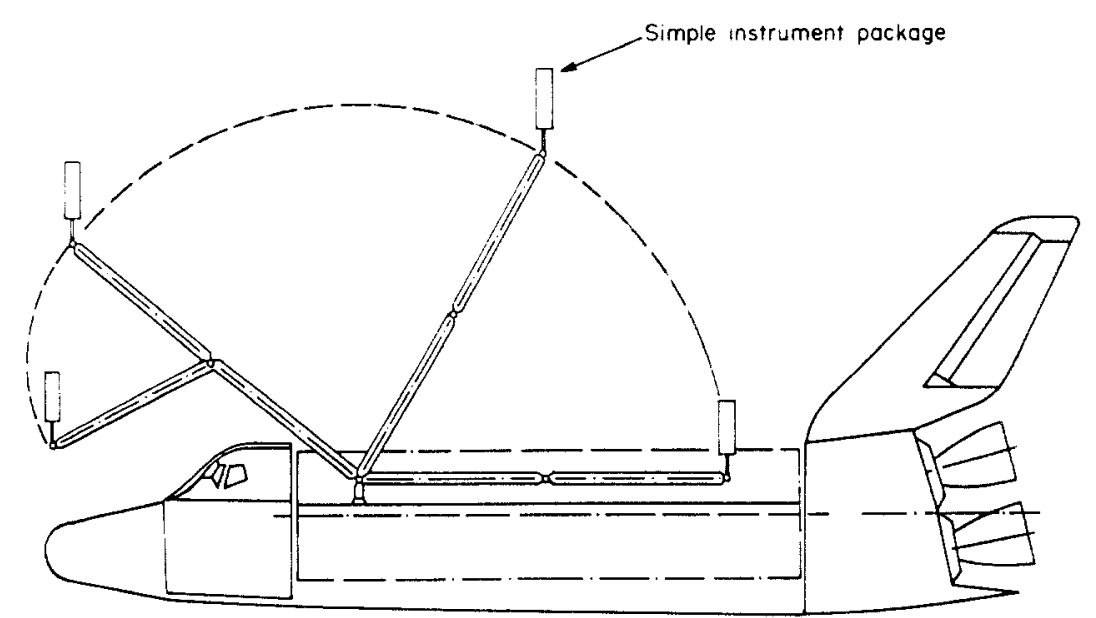

Fig. 20. The Spacelab/Orbiter with the RMS (extended in different ways) holding a simple instrument package. 


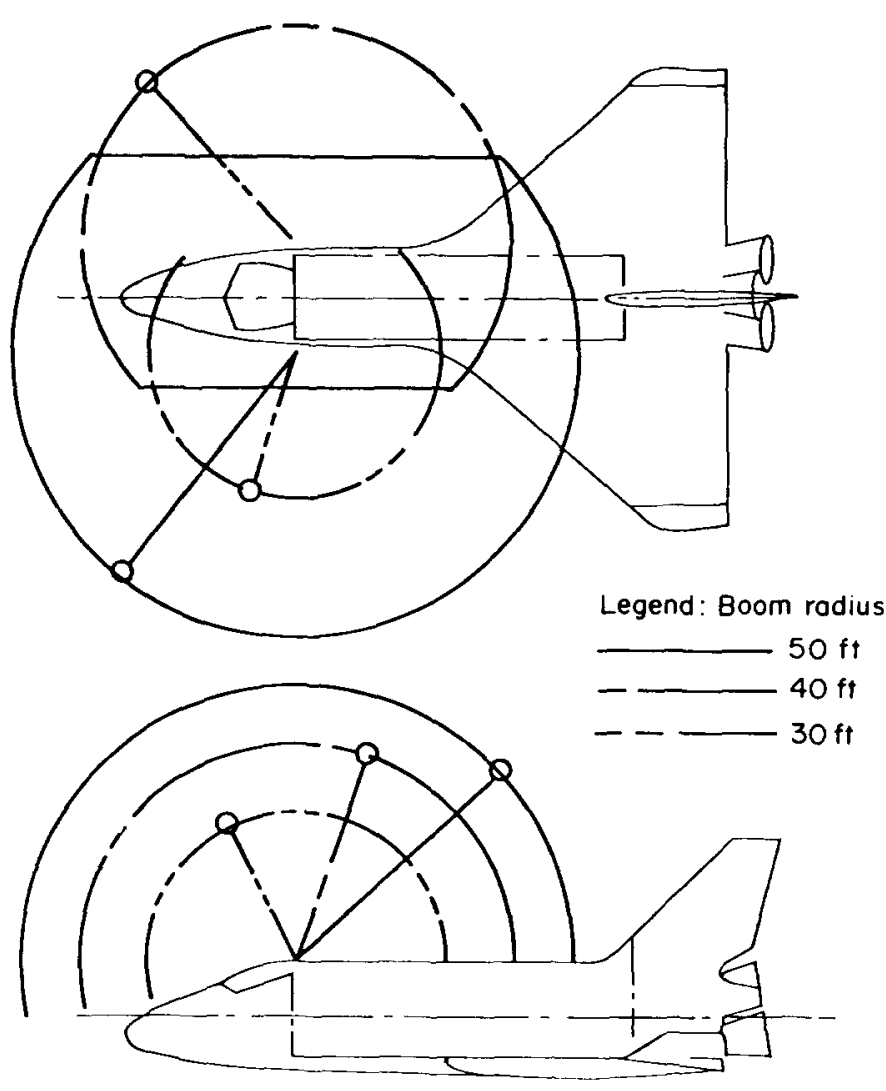

Fig. 21. The Spacelab/Orbiter with an attached TAD. The figure shows the limits of the region accessible to the TAD when boom (or: RMS) is extended to 30,40 and $50 \mathrm{ft}$ from surface.

The errors in position for this maneuver are primarily due to inaccuracies in orienting the Shuttle $\left( \pm 1^{\circ}\right)$ and the magnitude of thrust $( \pm 0.04 \mathrm{sec}$ of firing). The maximum possible error in position due to variations in differential drag were also analyzed, and it was found to be $0.25 \mathrm{~m}$ at the end of arc 1 which was small in comparison to errors due to inaccuracy in thruster magnitude and direction. Therefore, the errors due to variations in differential drag were ignored in the remaining calculations. These calculations were performed at NASA/Marshall Space Flight Center (Mullins, 1975).

Acknowledgements-The authors wish to acknowledge the interest and support of NASA's Office of Space Sciences and in particular the interest of Dr. E.R. Schmerling. Part of the work was supported by: NASA Grant NGR 23-005-320 and part by NSF Grant ATN 76-19790.

\section{References}

Al'pert Ya. L., Gurevich A. V. and Pitaevsky L. P. (1965) Space physics with artificial satellites (Monograph). Consultant Bureau. 
Al'pert Ya. L. (1976) Wave-like phenomena in the near-Earth plasma and interactions with manmade bodies. In Handbuch der Physik (Encyclopedia of Physics) (Edited by K. Rawer). Vol. $\mathrm{XL} 1 \mathrm{X} / 5$, p. 251. Geophysics III, part 5.

Andrijanov J. V. and Podgorny I. M. (1976) Effects of the frozen-in magnetic field on the formation of Venus' Plasma Shell Boundary. In Solar Wind Interactions with the Planets Mercury, Venus and Mars (Edited by N. F. Ness), p. 101. Proc. of a seminar of the U.S.A./U.S.S.R. Joint Working Group on Near Earth Space, the Moon and Planets. Moscow, November 1975.

Belotserkovskii O. M. and Mitnitskii V. Ya. (1976) Numerical study of some solar-wind interaction models with space objects. In Solar Wind Interactions with the Planets Mercury, Venus and Mars (Edited by N. F. Ness) p. 121. Proc. of a seminar of the U.S.A./U.S.S.R. Joint Working Group on Near Earth Space, the Moon and Planets. Moscow, November 1975.

Block L. P. (1967) Scaling considerations for magnetospheric model experiments Plan. Space Sci. 15. 1479-1487.

Block L. P. (1975) In Physics of the Hot Plasma in the Magnetosphere, p. 229.

Block L. P. (1976) Interpretation of laboratory experiments of interest to space physics. In Phys. of Plan. Environments (Edited by D. J. Williams), Vol. I, p. 255.

Block L. P. and Falthammar C. G. (1976) In Annales de Geophys., Vol. II 32; p. 1926,

Boyd R. L. F. (1968) in Plasma Diagnostics (Edited by W. Lochte-Holtgraven), p. 732. Wiley, New York and North-Holland, Amsterdam.

Brace L. H. and Findlay J. (1969) Comparison of cylindrical electrostatic probe measurements on Alouette II and Explorer 31 satellites. Proc. of I.E.E.E. 57(6), 1057-1060.

Brace L. H., Theis R. F. and Dalgarno A. (1973) The cylindrical electrostatic probe for AtmosphereExplorer C, D and E satellites. Radio Science 8(4), 34-348.

Call S. M. (1969) The interaction of a satellite with the ionosphere. Rep. 46, Plasma Laboratory, Columbia University.

Cloutier P. A. (1976) Solar-wind interactions with planetary ionospheres. In Solar Wind Interactions with the Planets Mercury, Venus and Mars. (Edited by N.F. Ness), p. 111. Proc. of a seminar of the U.S.A./U.S.S.R. Joint Working Group on Near Earth Space, the Moon and Planets. Moscow, November 1975.

Danielson L. R. and Kasai G. H. (1968) Laboratory simulation of plasma phenomena in comets. $J$. Geophys. Res. 73, 259-266.

Dimant Y.S. and Gurevich A. V. (1975) Flow of a Rarefied Plasma Around a Disc. Geomagnetism and Aeronomy 15(2), 183-190.

Donley J. L. (1969) The thermal ion and electron trap experiments on the Explorer 31 satellite. Proc. of I.E.E.E. 57(6), 1061-1063.

Dryer M. (1970) Solar wind interactions-Hypersonic analogue. Cosmic Electrodynamics 1, 115-142.

Dubinin E. M. and Podgorny I. M. (1974) Particle precipitation and radiation belt in laboratory experiments. J. Geophys. Res. 79, 1426-1431.

Dubinin E. M, Podgorny I. M., Potanin Yu. M. and Sonnet C. P. (1977) Laboratory Simulation of the Lunar magnetosphere. Geophys. Res. Lett. 4(10), 391-394.

Fahleson U. (1967) Laboratory experiments with plasma flow past unmagnetized obstacles. Plan. Space Sci. 15, 1489-1497.

Falthammar C. G. (1974) Laboratory experiments of magnetosphere interest. Space Sci. Rev. 15, 803-825.

Findlay J. and Brace L. H. (1969) The cylindrical electrostatic probes employed on Alouette II and Explorer 31 satellites. Proc. of I.E.E.E. 57(6), 1054-1056.

Fournier G. (1974) Ecoulment de plasma sans collisions aurout d'un cylindre en vue d'applications aux sondes ionospheriques. Office National d'Etudes et de Rech. Aerosp.-ONERA Chatillon, France, Public. 137.

Fournier G. and Pigache D. (1975) Wakes in collisionless plasmas. In Physics of Fluids, Vol. 18, p. 1443-1453.

Fournier G. and Pigache D. (1977) Etude de plasmas de synthese pour la simulation du vol ionospherique. Rev. Physique Appl. t. 12, p. 1063-1070.

Goddard R. (1975) A symmetrical model for cylindrical and spherical collectors in a flowing collisionless plasma. Ph.D. Thesis. York University, Toronto, Canada. 
Grabowski R. and Fischer T. (1975) Theoretical density distribution of plasma streaming around a cylinder. Plan. Space Sci. 23, 287-304.

Gurevich A. V. (1962) Perturbations in the ionosphere caused by a traveling body. Planet Space Sci.9, 321-344.

Gurevich A. V., Pitaevski L. P. and Smirnova V. V. (1970) Ionospheric aerodynamics. SovietPhysics-Uspekhi 99, 595-619.

Henderson C. L. and Samir U. (1967) Observation of the disturbed region around an ionospheric spacecraft. Plan. Space Sci. 15, 1499-1513.

Hester S. D. and Sonin A. A. (1970) A laboratory study of the wakes of ionospheric satellites. AIAA J. 8, 1090-1098.

Illiano J. M. and Storey L. R. O. (1974) Apparent enhancement of electron temperature in wake of a spherical probe in a flowing plasma. Plan. Space Sci. 22, 873-878.

Isensee U. (1977) Plasma disturbances caused by the Helios spacecraft in the solar wind. J. Geophys. 42, 581-591.

Kasha M. A. (1969) (Monograph): The Ionosphere and its Interaction with Satellites. Gordon \& Breach, Science Publication.

Kawashima N. (1971) Characteristics of intruding plasma in a simulated magnetosphere. In Cosmic Electrodynamics 1, 415-427.

Kristopherson L. (1975) Tech. Rep. TRITA-EPP, 75-12, Dept. of Plasma Physics, Royal Institute of Tech. Stockholm, Sweden.

Kunemann B. (1978) The collisionless flow of unmagnetized plasmas around bodies. J. Plasma Phys. 20(1), 17-30.

Lipatov A. S. (1976) Numerical simulation of the effects of magnetic fields induced by plasma flow past non-magnetic planets. In Solar Wind Interactions with the Planets Mercury, Venus and Mars (Edited by N. F. Ness), p. 151. Proc. of a seminar of the U.S.A./U.S.S.R. Joint Working Group on Near Earth Space, the Moon and Planets. Moscow, November 1975.

Liu V. C. (1969) Ionospheric gas dynamics of satellites and diagnostic probes. Space Sci. Rev. 9, $423-490$.

Liu V. C. (1975) On ionospheric aerodynamics. Progress in Aerospace Science 16, 273-297.

Maier E. J. R. (1969) Explorer 31 total current monitor experiments. Proc. of I.E.E.E. 57(6), 1068-1071.

Martin A. R. (1974) Numerical solutions to the problem of charged particle flow around ionospheric spacecraft. Plan. Space Sci. 22, 121-141.

Maslenikov M. S. and Sigov Yu. S. (1968) Numerical experiments on flow of rarefied plasma around bodies of various shapes. Cosmic Res. 6, 184-190.

Miller N. J. (1972) Some implications of satellite spin effects in cylindrical probe measurements. J.G.R. 77(16), 2851-2861.

Mullins L. D. (1975) The motion of throw-away detectors (TAKs) relative to the Space Shuttle. NASA-TMX/64948.

Ohyabu N., Okamura S. and Kawashima N. (1973) Preprint of the Univ. of Tokyo/Institute of Space and Aeronautical Sciences.

Olson W. P. (1974) Potential experiments with superconducting magnets in Earth orbit. Space Sci. Rev. 15, 899-904.

Oran W. A., Samir U. and Stone N. A. (1974) Parametric study of the near-wake structure of spherical and cylindrical bodies in the laboratory. Planet. Space Sci. 22, 379-390.

Oran W. A., Stone N. H. and Samir U. (1975) The effects of body geometry on the structure in the near wake zone of bodies in a flowing plasma. J. Geophys. Res. 80, 207-209.

Parker L. W. (1976) Computation of collisionless steady state plasma flow past a charged disc. NASA Rep. CR-144159.

Parker L. W. (1977) Calculation of sheath and wake structure about a pillbox-shaped spacecraft in a flowing plasma, p. 331. In Proc. of the Spacecraft Charging Technology Conf. AFGL-TR-77. 0051 .

Perez-de-Tejada H., Dryer M. and Vaisberg O. L. (1977) Viscous flow in the near-Venusian plasma wake. J. Geophys. Res. 82, 2837-2841.

Pike C. P. and Lovell R. R. (1977) (Editors) Proc. Spacecraft Charging Technology Conf. AFGL-TR77-0051: NASA TMX-73537. 
Podgorny I. M. and Sagdeev R. Z. (1970) Physics of the inter-planetary plasma and laboratory simulation. Soviet-Physics/Uspekhi 98, 445-462.

Podgorny I. M., Andrijanov Yu. V. and Dubinin E. M. (1973) Electric field micro-fluctuations on the shock front in model experiments. Astrophys. Space Sci. 24, 245-257.

Podgorny I. M. and Dubinin E. M. (1974) Laboratory experiments directed toward the investigation of magnetospheric phenomena. Space Sci. Rev. 15, 827-840.

Podgorny I. M. (1976) Laboratory experiments (plasma intrusion into the magnetic field). In Phys. of Plan. Environments (Edited by D. J. Williams), Vol. 1, p. 241.

Rosen A. (1976) (Editor) Spacecraft charging by magnetospheric plasmas. Prog. in Astronaut. Aeronaut. 47.

Rupp C. C. and Laue S. H. (1978) Proc. of the 1978 Goddard Mem. Symp. No. 78-048. Washington, D. C.

Samir U. and Willmore A. P. (1965) The distribution of charged particles near a moving spacecraft. Plan. Space Sci. 13, 285-296.

Samir U. and Willmore A. P. (1966) The equilibrium potential of a spacecraft in the ionosphere. Plan. Space Sci. 14, 1131-1137.

Samir U. and Wrenn G. L. (1969) The dependence of charge and potential distribution around a spacecraft on ionic composition. Plan. Space Sci. 17, 693-706.

Samir U. and Wrenn G. L. (1972) Experimental evidence of an electron temperature enhancement in the wake of an ionospheric satellite. Plan. Space Sci. 20, 899-904.

Samir U. and Jew H. (1972) Comparison of theory with experiment for electron density distribution in the near wake of an ionospheric satellite. J. Geophys. Res. 77, 6819-6827.

Samir U. Maier E. J. R. and Troy B. E. Jr. (1973) The angular distribution of ion flux around an ionospheric satellite. J. Atmos. Terrest. Phys. 35, 513-519.

Samir U. (1973a) Charged particle distribution in the nearest vicinity of ionospheric satellitescomparison of the main results from the Ariel I, Explorer 31 and Gemini-Agena 10 Spacecraft. Photon and Particle Interactions with Surfaces in Space (Edited by R. J. L. Grard), pp. 193-219. Reidel, Dordrecht, Netherlands.

Samir U. (1973b) Large space vehicles-Platforms for second generation in situ wake observations. J. Astron. Sci. 20, 347.

Samir U., Oran W. A. and Stone N. H. (1974a) Laboratory simulation of space aerodynamic phenomena: satellite wake studies. In Rarefied Gas Dynamics, p. D11. 9th Inter. Symp.

Samir U., Stone N. H. and Oran W. A. (1974b) Does a two-beam flow describe structural patterns in the wake of large bodies? Astrophys. Space Sci. 31, L1.

Samir U., First M., Maier E. J. and Troy B. E. (1975) A comparison of the Gurevich et al. and the Liu-Jew wake models for the ion flux around a satellite. J. Atmos. and Terr. Phys. 37, 557-586.

Samir U. (1976) Space related laboratory experiments-rarefied plasma flows over bodies. EOSTrans. Amer. Geophys. Union 56, 1032.

Samir U., Carignan G. R., Stone N. H., Oran W. A. and Parker L. W. (1976) An investigation of the induced charged particle environment near the Spacelab. Proposal to NASA.

Samir U., Stone N. H. and Oran W. A. (1977) Ionospheric aerodynamics and space simulation studies utilizing the Shuttle/Spacelab platform. Rarefied Gas Dynamics (Edited by J. L. Potter), Vol. 51, Part 2, p. 975. Prog. in Astronautics and Aeronautics Series.

Samir U., Gordon R., Brace L. H. and Theis R. F. (1979a) The near-wake structure of the Atmosphere Explorer C (AE-C) satellite-a parametric investigation. J. Geophys. Res. 84(A.2), 513-525.

Samir U., Brace L. H. and Brinton H. C. (1979b) About the influence of electron temperature and relative ionic composition on ion depletion in the wake of the AE-C satellite. Geophys. Res. Lett. 6(2), 101-104.

Schatzman E. and Bierman L. (1974) Cosmic Gas Dynamics (Edited by M. S. Uberoi).

Schindler K. (1969) Laboratory experiments related to the solar wind and the magnetosphere. In Magnetospheric Physics (Edited by D. J. Williams and G. Mead), p. 69, 1969. Also in J. Geophys. Res. 7(1-2), 51-75.

Sprieter J. R. and Rizzi A. W. (1972) The Martian bow wave theory and observation. Planet. Space Sci. 20, 205-208. 
Stone N. H., Samir U. and Oran W. A. (1974) Laboratory simulation of the structure of disturbed zones around bodies in space. J. Atmos. Terrest. Phys. 36, 253-260.

Stone N. H., Samir U. and Wright K. H. Jr (1978) Plasma disturbances created by probes in the ionosphere and their potential impact on low energy measurements considered for Spacelab. $J$. Geophys. Res. 83, 1668-1672.

Stone N. H. and Samir U. (1978) The tethered subsatellite as a tool for large body plasma flow interaction studies. In Proc. UAH/NASA Workshop on the Uses of a Tethered Satellite System, pp. 182-194. Huntsville, Alabama, May 1978.

Stone N. H. and Samir U. (1980) Bodies in flowing plasmas: Laboratory studies. Presented COSPAR XXIII, Budapest, Hungary, 2-14 June 1980. To be published in Advances in Space Exploration.

Troy B. E. Jr., Maier E. J. and Samir U. (1975) Electron temperatures in the wake of an ionospheric satellite. J. Geophys. Res. 80, 993-997.

Vernet-Mayer N. (1976) Rocket spin effects on the current collected by a cylindrical probe in the ionosphere. J. Geophys. Res. 81, 450-456.

Wallis M. K. (1973) Weakly-shocked flows of the solar wind plasma through atmospheres of comets and planets. Planet. Space Sci. 21, 1647-1660.

Weil H., Jew H. and Samir U. (1973) Structure of the ionospheric disturbances about planetary entry probes. In Photon and Particle Interactions in Space (Edited by R. J. L. Garard), pp. 101-126. Reidel, Dordrecht-Holland.

Whipple E. C. Jr. (1965) The equilibrium electric potential of a body in the upper atmosphere and in interplanetary space. NASA Rep. X-615-65-296, G.S.F.C.

Whipple E. C. (1977) Modeling of spacecraft charging. In Proc. of the Spacecraft Charging Technology Conf. (Edited by Pike and Lovell), p. 225.

Williamson P. R. and Banks P. M. (1976) The tethered balloon current generator-a Space Shuttle-tethered subsatellite for plasma studies and power generation. NOAA Contract Rep. 03-5-022-60.

Wu S. T. and Dryer M. (1973) Kinetic theory analysis of solar wind interaction with planetary objects. In Photon and Particle Interactions with Surface in Space, pp. 453-470. Rzidel, Holland. 